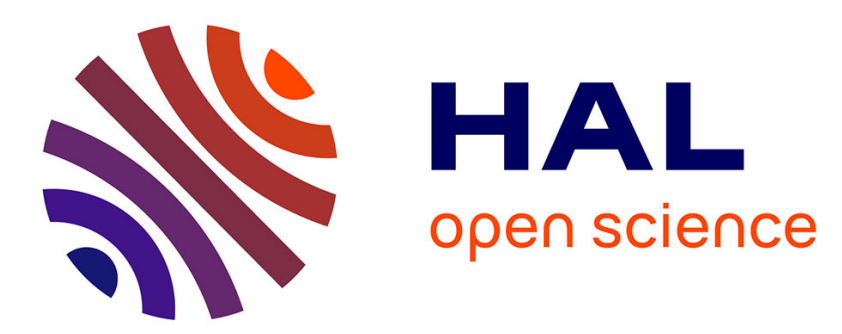

\title{
Procédure d'identification inclusive d'un système thermique. Etude de cas : caractérisation d'un capteur solaire à air en régime dynamique \\ J.-L. Dufresne, A. Lahellec, L.-M. Chounet
}

\section{- To cite this version:}

J.-L. Dufresne, A. Lahellec, L.-M. Chounet. Procédure d'identification inclusive d'un système thermique. Etude de cas: caractérisation d'un capteur solaire à air en régime dynamique. Revue de Physique Appliquée, 1990, 25 (11), pp.1139-1160. 10.1051/rphysap:0199000250110113900 . jpa00246284

\section{HAL Id: jpa-00246284 \\ https://hal.science/jpa-00246284}

Submitted on 1 Jan 1990

HAL is a multi-disciplinary open access archive for the deposit and dissemination of scientific research documents, whether they are published or not. The documents may come from teaching and research institutions in France or abroad, or from public or private research centers.
L'archive ouverte pluridisciplinaire HAL, est destinée au dépôt et à la diffusion de documents scientifiques de niveau recherche, publiés ou non, émanant des établissements d'enseignement et de recherche français ou étrangers, des laboratoires publics ou privés. 
Classification

Physics Abstracts

$06.50-86.10 \mathrm{~K}$

\title{
Procédure d'identification inclusive d'un système thermique. Etude de cas : caractérisation d'un capteur solaire à air en régime dynamique
}

\author{
J.-L. Dufresne, A. Lahellec et L.-M. Chounet \\ Equipe RAMSES (UPR 293 du CNRS), Centre Universitaire, Bât. 508, 91405 Orsay Cedex, France
}

(Reçu le 9 mai 1989, révisé le 17 mai 1990, accepté le 23 juillet 1990)

\begin{abstract}
Résumé. - Une étude de cas concernant la caractérisation en régime dynamique d'un composant solaire de bâtiment nous a permis d'aborder le problème général que soulève l'identification d'un modèle physique, non linéaire, à nombre élevé de paramètres libres (ici dix). Le point de vue retenu est que le modèle à construire et à déterminer a trois objectifs couplés : apporter au concepteur une compréhension suffisante du fonctionnement du composant afin de guider ses tentatives d'améliorations, autoriser son intégration dans un système plus vaste (modèle de bâtiment et d'organes de commande) afin d'éprouver ses performances en situation réelle, et enfin guider le choix de séquences expérimentales pour répondre aux mieux aux deux objectifs précédents (cohérence globale). On décrit la méthode, sa mise en œuvre, et on donne les résultats obtenus en mettant en évidence la cohérence explicite qui est établie entre la phase expérimentale - effectuée dans un contexte de normalisation par un centre technique - et la détermination proprement dite des grandeurs physiques du modèle. Contrairement aux méthodes classiques de mesure pour lesquelles on peut concevoir des expériences découplant chacun des paramètres, la procédure choisie identifie d'un coup l'ensemble des paramètres libres sur l'ensemble des données expérimentales (identification inclusive). Un accent particulier est mis sur l'analyse des erreurs de mesure dont l'importance est manifeste, non seulement pour déterminer la précision du modèle obtenu, mais également pour utiliser correctement l'ensemble de l'information expérimentale pour la phase d'identification proprement dite.
\end{abstract}

\begin{abstract}
The dynamical characterisation of a solar component - taken as a case study - introduced us to the general problem of non linear multiparametric model identification (10 parameters in our case). The elaboration of such a model has three goals : first, it has to allow the designer to understand and improve his components; then it can be integrated in a global"system model (the building and control devices) for real performances study; and finally, it is a guidance for the monitoring process in the purpose of fulfilling the two previous objectives (global coherence). The method and its implementation are described, and the results obtained for our case study given; we set a particular emphasis on the embedded coherence between the monitoring stage - performed in a " Technical Center " in the context of a normalisation procedure - and the physical model parameters identification. In opposition to classical measurement operation which allow different experiments to decouple each parameter, the described method determines at once the whole set of parameters versus the whole set of available experimental data (inclusive identification). We then display a few procedure intrinsic coherence tests as well as the accuracy obtained on the determined parameters. One of the critical point of the procedure is the measurement error analysis, because of its imporance not only for the resulting model accuracy, but as an essential information for a proper use of the experimental ata in $t e$ identification procedure itself.
\end{abstract}

\section{Nomenclature.}

$b \quad$ Paramètre multiplicatif dans la loi du coefficient d'échange convectif absorbeur-fluide arrière et peaufluide arrière.

$d, e$ Paramètres du coefficient d'échange convectif vitre-fluide avant et absorbeur-fluide avant. Exposant de Re dans la loi du coefficient d'échange convectif absorbeur-fluide arrière et peau-fluide arrière. 
$q_{j} \quad$ Ecart des puissances mesurées-calculées pondéré par les erreurs de mesures, à l'instant $j$.

$A \quad$ Matrice des dérivées secondes de $Q^{2}$ selon les paramètres (dimension $K \times K$ ).

$C \quad$ Capacité thermique par unité de surface du capteur.

$D_{0} \quad$ Diamètre des tuyaux de l'échangeur analogue.

$D$ Diamètre hydraulique dans l'enceinte avant du capteur.

$H$ Conductances thermiques.

$K \quad$ Nombre de paramètres libres.

$L \quad$ Longueur (dans le sens de l'écoulement) des parois.

$|M\rangle$ Vecteur des puissances extraites calculées, de dimension $N$.

$M_{j} \quad$ Puissance extraite calculée à l'instant $j$.

$N \quad$ Nombre d'événements de mesure utilisés pour l'ajustement.

$P \quad$ Puissances thermiques par unité de surface de capteur, pour une tranche de capteur.

$Q^{2} \quad$ Somme, sur l'ensemble des points expérimentaux, des écarts quadratiques pondérés mesure-modèle $q_{j}^{2}$. Grandeur à minimiser par la procédure d'ajustement.

$R \quad$ Résistance thermique $(1 / H)$.

$T \quad$ Température d'un nœud.

$V \quad$ Matrice de covariance des erreurs de mesure (dimension $N \times N$ ).

Pr Nombre de Prandtl.

$\operatorname{Re}$ Nombre de Reynolds.

$\alpha \quad$ Coefficient d'absorption.

$\varepsilon_{j} \quad$ Erreur, à l'instant $j$, sur la différence entre les puissances extraites mesurée et calculée, due aux erreurs de mesures des différentes grandeurs.

$\lambda \quad$ Conductivité thermique de l'air.

$|\mu\rangle$ Vecteur des puissances extraites mesurées (dimension $N$ ).

$\mu_{j} \quad$ Puissance extraite mesurée à l'instant $j$.

$|\omega\rangle$ Vecteurs des paramètres.

II Matrice de covariance des paramètres libres (dimension $K \times K$ ).

INDICES SUPÉRIEURS; TYPE DE TRANSFERT.

c Echange thermique par convection.

$\mathrm{k} \quad$ Echange thermique par conduction.

$\mathrm{r}$ Echange thermique par rayonnement.

INDICES INFÉRIEURS; COMPOSANTS ET ENVIRONNEMENT.

a Absorbeur.

ar Local arrière.

c «ciel » (échange radiatif grande longueur d'onde).

e Air extérieur, devant le capteur.

f Air caloporteur circulant entre l'absorbeur et le fond (fluide arrière).

g Air caloporteur circulant entre la vitre et l'absorbeur (fluide avant).

i Entrée.

$j \quad$ Evénement (ou instant) de mesure $j$.

1 Isolant du fond.

o Sortie.

p Peau (ou surface intérieure) de l'isolant arrière.

v Vitre.

\section{Introduction.}

Les outils d'analyse thermique du bâtiment de nouvelle génération, tels que ceux qui font l'objet du "Groupement d'Etude et de Recherche Almeth ${ }^{(1)}$ » [1], doivent notamment permettre à un indus-

(') Atelier-Logiciel pour la Maîtrise de l'Energie dans le Tertiaire et l'Habitat ; groupe de recherche incluant des équipes du CNRS, du CSTB, de l'EMP, de l'ENPC, de la FNB et de l'UPS, sous l'égide de l'AFME. triel d'éprouver par simulation un composant placé dans son environnement réel. Grâce à une forte modularité, l'utilisateur peut inclure simplement un modèle de composant innovant, et agréger autour de lui les modèles correspondant au bâtiment et à son environnement. On dispose alors d'un outil complet permettant d'évaluer des performances, d'essayer diverses améliorations, d'introduire des lois de commandes variées, etc.

Le travail qui fait l'objet de cet article porte sur la conception d'un modèle thermique rentrant dans ce 
cadre et prenant en compte les possibilités de sa détermination expérimentale.

La méthode proposée a tenté de donner une cohérence maximale entre les objectifs de l'industriel, la définition d'un modèle adapté et la mise en œuvre d'une procédure expérimentale appropriée. Elle a été utilisée avec succès pour caractériser un composant de façade intégrant un capteur solaire à air [2].

La cohérence de la démarche repose sur la procédure d'identification paramétrique choisie, qui est à même d'effectuer rigoureusement l'articulation entre les objectifs d'utilisation du modèle, le modèle luimême et les séquences expérimentales.

Nous présentons dans cet article la procédure d'identification elle-même, les aspects expérimentaux, ainsi que la mise en œuvre pour les applications industrielles ayant déjà été présentée [3].

Nous expliciterons tout d'abord (paragraphe 2) le problème tel qu'il se pose, puis nous présenterons le composant étudié ainsi que la modélisation proposée (paragraphe 3). Après avoir décrit la méthode d'ajustement des paramètres (paragraphe 4) et l'expérimentation réalisée (paragraphe 5), nous aborderons en détail la mise en œuvre de la procédure d'ajustement (paragraphe 6). L'analyse critique des résultats (paragraphe 7) nous permettra enfin de vérifier la cohérence de l'ensemble de la procédure d'identification (modélisation - expérimentation ajustement des paramètres).

Il nous a semblé plus clair de faire le point en fin d'article sur l'originalité de l'application de la méthode décrite par rapport à divers travaux concernant l'automatique, le génie chimique, et la thermique.

\section{Exposé du problème.}

2.1 OBJECTIFS FINAUX. - Nous nous plaçons dans le cas d'un concepteur ayant l'ambition d'améliorer les performances de composants mur-capteur solaire en recherchant leur efficacité en situation réelle. Il fait appel par exemple à un Centre Technique pour lui fournir des résultats d'essai ; c'est d'ailleurs dans le cadre du développement d'une méthode d'essais normalisés pour les capteurs solaires à air que s'est situé historiquement notre travail, dont la partie en 84-87.

Ainsi, la grandeur importante est la puissance calorifique extraite du capteur. De plus, le modèle thermique doit être physiquement interprétable si l'on veut analyser les résultats pour une recherche d'amélioration des performances. Enfin, il est souhaitable que la précision du modèle fourni soit donnée, faute de quoi son utilisation ultérieure dans

( $\left.{ }^{2}\right)$ CETIAT : Centre Technique des Industries Aérauliques et Thermiques. des outils complets de simulation risque d'être aberrante.

2.2 DÉfinition D'UN MODÈle ADAPTÉ. - Pour modéliser un système physiquement complexe comme celui de notre étude, deux solutions extrêmes sont couramment adoptées :

- ou bien l'on connaît les lois phénoménologiques locales et l'on est capable d'utiliser les outils du calcul numérique pour les articuler sur l'ensemble du système (modèle dit détaillé) ;

- ou bien l'on renonce à toute phénoménologie et l'on introduit un modèle mathématique global faisant le lien entre les grandeurs d'entrée et de sortie (modèle dit " boîte noire »).

Pour établir un modèle détaillé, on se base sur des lois locales. Or, pour des systèmes physiquement complexes, ces lois peuvent être mal connues ou leur intégration numérique très délicate. Enfin, ces modèles sont lourds à utiliser; leur étalonnage et leur validation expérimentale sont difficiles.

A l'inverse, les modèles « boîtes noires », s'ils sont opérationnels, n'ont pas de valeur explicative intrinsèque et leur utilisation dans un contexte différent des conditions d'étalonnage est risquée.

L'approche par modèle adapté est intermédiaire (modèle « boîte grise » [4]), en ce qu'elle repose sur une formulation physique globale relativement simple. Les lois utilisées sont conformes à l'expression des lois phénoménologiques locales, mais ces lois étant maintenant globalisées, leurs paramètres doivent être déterminés expérimentalement. On peut résumer cette démarche en disant que l'ajustement expérimental de grandeurs globales se substitue à l'intégration numérique des lois locales.

2.3 NÉCESSITÉ D'UN AJUSTEMENT GLOBAL DES PARAMÈTRES. - Le processus d'identification est, de façon générale, constitué des étapes suivantes :

- définition d'un modèle adapté ;

- définition d'une expérimentation ;

- choix des paramètres libres et ajustement;

- détermination de la précision des résultats obtenus et de la pertinence du modèle ;

- vérification de la pertinence de l'ensemble de la démarche.

tion quasi-réelle, il n'y a généralement pas de séquences expérimentales permettant d'isoler totalement l'influence de quelques paramètres par rapport aux autres. Nous disposons alors, d'une part d'un modèle non étalonné mais satisfaisant a priori et, d'autre part, d'un grand nombre de mesures pour des expériences ne découplant que partiellement les paramètres. La méthode d'identification ne peut plus être heuristique. Elle doit utiliser dans son ensemble le domaine de mesure $\{|\boldsymbol{\mu}\rangle\}$ pourvu d'une métrique choisie en fonction de l'incertitude 
sur chacune des grandeurs mesurées, pour déterminer l'ensemble des paramètres $\{|\omega\rangle\}$. C'est cette méthode, couramment utilisée pour l'analyse de données dans certaines disciplines - physique des particules [5], physique du solide [6]... - que nous appliquons ici à l'analyse thermique d'un système.

\section{Modélisation du système étudié.}

3.1 Présentation du MUR-CAPTEUR SOlAire. Le capteur solaire-plan à air dont nous présentons ici l'étude d'un prototype (capteur "ACRET») (Fig. 1) a été conçu comme composant de façade. Positionné verticalement, ce capteur est autoporteur et intègre les 3 fonctions suivantes : capteur solaire, mur extérieur et isolant de façade. Le fluide caloporteur est de l'air circulant entre le fond et l'absorbeur, tous deux de forme ondulée afin d'améliorer les échanges convectifs parois-fluide. Une vitre est située devant l'absorbeur pour diminuer les pertes thermiques (" effet de serre »).
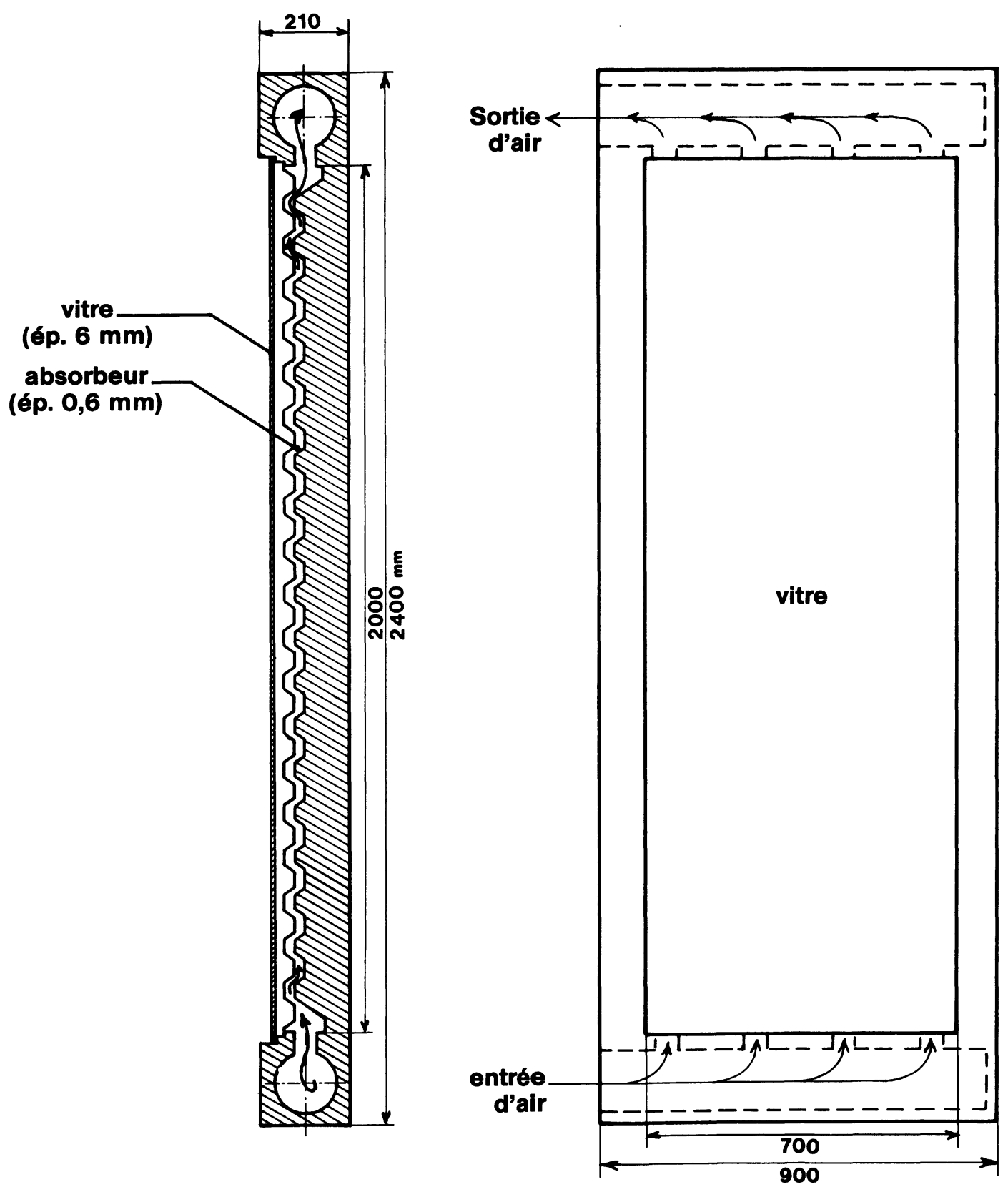

Fig. 1. - Coupe longitudinale et vue de face du capteur solaire à air "ACRET».

[Longitudinal cross-section and front view of the «ACRET » air solar collector.] 

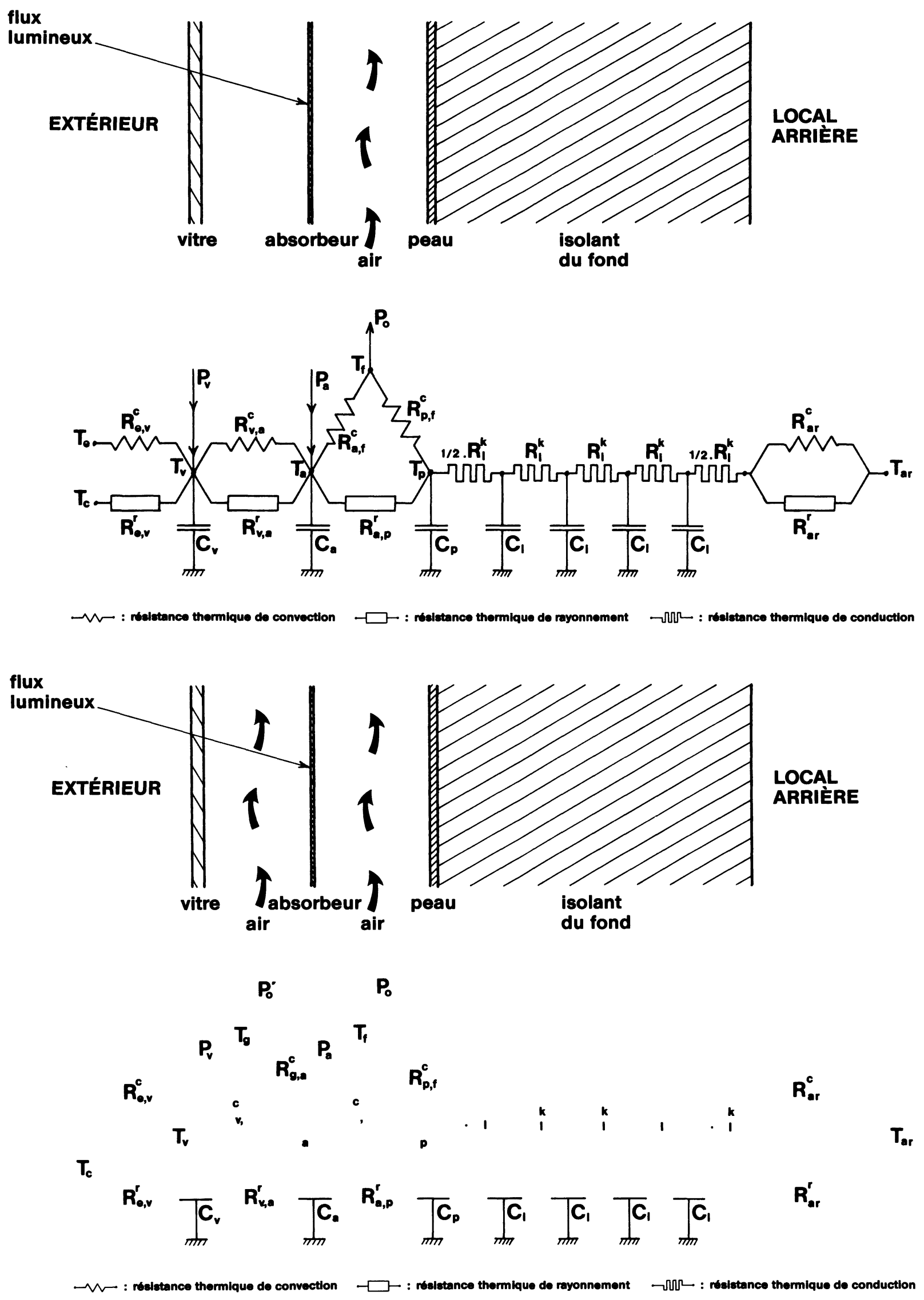

Fig. 2. - a) Coupe schématique d'un capteur solaire à enceinte avant figée et discrétisation nodale correspondante. b) Idem en a), mais avec une circulation parasite d'air entre la vitre et l'absorbeur.

[a) Cross-section of an air solar collector, and attached lumped model. b) Same as a), but with a parasite air flow rate between the glazing and the absorber-plate.] 
3.2 CHOIX D'UN MODÈLE ADAPTÉ. - Tirant parti des différents travaux précédents réalisés sur les capteurs solaires à air [7-11], nous avons établi un modèle qui répond a priori aux objectifs de notre étude : bles ;

- les paramètres sont physiquement interpréta-

- les régimes dynamiques et le fonctionnement à débit variable sont bien décrits.

Ce modèle est quasi-bidimensionnel : il est constitué d'un assemblage en série de plusieurs modèles élémentaires, monodimensionnels, basés sur la discrétisation nodale d'une "tranche" simplifiée de capteur. Une coupe schématique du capteur étudié et le modèle monodimensionnel correspondant sont représentés figure $2 \mathrm{a}$ (capteur à enceinte avant figée). Comme nous le verrons plus loin, nous avons été amenés à supposer l'existence d'une circulation parasite d'air entre l'absorbeur et la vitre. Nous présentons dès à présent le modèle élémentaire que nous avons alors retenu (Fig. 2b).

Principales hypothèses physiques de ce modèle:

- l'écoulement est purement longitudinal ;

- la loi d'échange solide-fluide est la même en tout point ;

- les effets de pertes latérales sont négligeables;

- la conduction longitudinale entre les sections des différents composants du capteur (vitre, absorbeur, fond) est négligeable.

Les sept grandeurs physiques d'entrée sont :

- le flux solaire ;

- la température de l'air devant le capteur;

- la vitesse de l'air devant le capteur (vent);

- la température du filtre atmosphérique (« température de ciel »);

- la température ambiante derrière le capteur ;
- le débit d'air dans le capteur;

- la température de l'air entrant dans le capteur.

En résolvant simultanément les équations de ce modèle analogique, on obtient directement l'expression de la température de chacun des nœuds en fonction des grandeurs d'entrée. Le nœud correspondant à la température de sortie d'air permet de calculer la puissance extraite du capteur, utilisée lors de l'identification.

3.3 EXPRESSION DES LOIS D'ÉCHANGES. - Pour modéliser les échanges convectifs absorbeur-fluide arrière et peau-fluide arrière, nous avons fait l'analogie avec un échangeur à tubes ovales disposés en quinconce (Fig. 3). Nous prenons alors la loi d'échange donnée par Mac Adams lorsque le régime est turbulent [12] :

$$
H_{\mathrm{a}, \mathrm{f}}^{\mathrm{c}}=H_{\mathrm{p}, \mathrm{f}}^{\mathrm{c}}=\lambda / D_{0} \cdot b_{0} \cdot \mathrm{Re}^{n}
$$

le nombre de Reynolds (Re) étant calculé par rapport au diamètre $D_{0}$, et $b_{0}$ et $n$ étant des constantes, valant respectivement 0,3 et 0,6 pour l'air.

Pour une meilleure lisibilité physique des résultats et pour mieux découper les paramètres $b_{0}$ et $n$, nous avons mis ce coefficient d'échange sous la forme :

$$
H_{\mathrm{a}, \mathrm{f}}^{\mathrm{c}}=H_{\mathrm{p}, \mathrm{f}}^{\mathrm{c}}=\left[b \cdot \operatorname{Re}_{120}^{(0,6-n)}\right] \cdot\left[b_{0} \cdot \lambda / D_{0} \cdot \operatorname{Re}^{n}\right]
$$

avec $\operatorname{Re}_{120}$, valeur de $\operatorname{Re}$ pour une température d'air de $40{ }^{\circ} \mathrm{C}$ et un débit de $120 \mathrm{~m}^{3} / \mathrm{h}$ (température et débit moyen de nos expériences). Les paramètres $b$ et $n$ ont pour le modèle référencé une valeur de 1 et 0,6 respectivement.

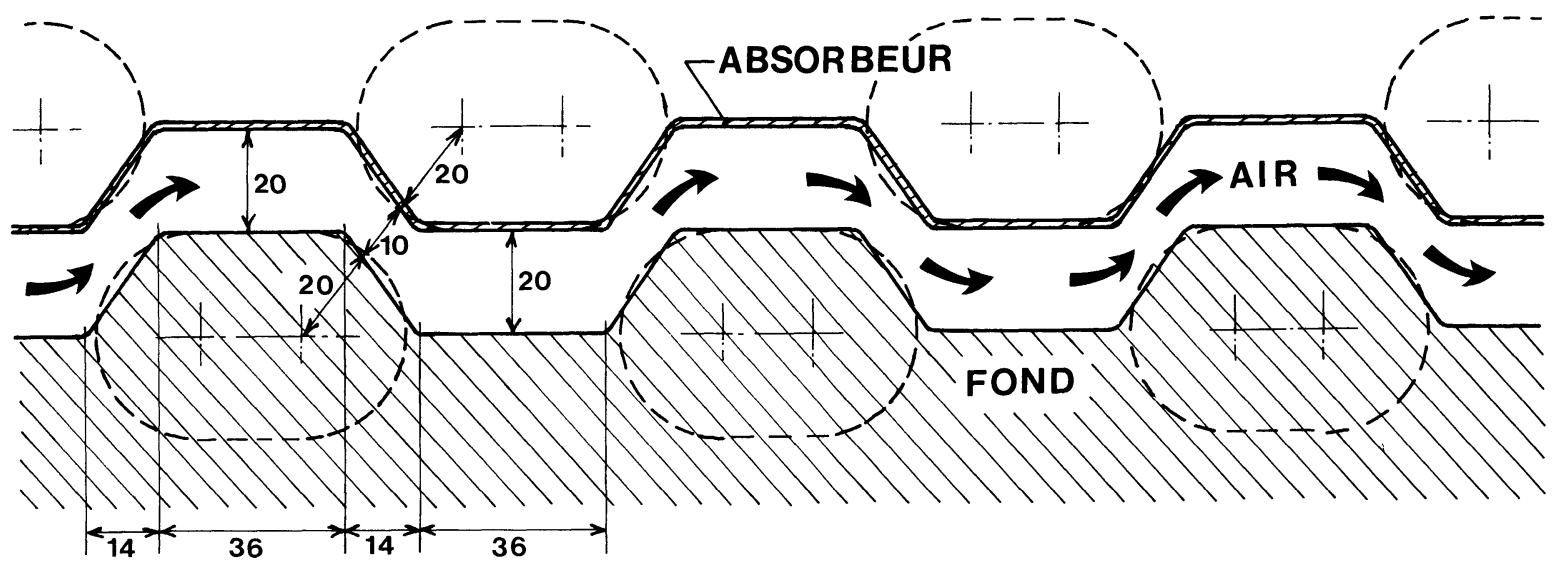

Fig. 3. - Coupe longitudinale du capteur «ACRET» (détail) et représentation - en pointillés - de l'échangeur à tubes avec lequel nous avons fait l'analogie pour estimer le coefficient d'échange convectif paroi-fluide arrière.

[Analogy between the real plate and it's equivalent model (oblong tubular heat exchangeur, in dotted line), for evaluation of the rear fluid-plate heat transfer coefficient.] 
En ce qui concerne les échanges convectifs absorbeur-fluide et vitre-fluide dans l'enceinte avant, nous avons choisi - après la mise en évidence de la circulation parasite d'air - l'expression suivante [13] :

$$
H_{\mathrm{v}, \mathrm{g}}^{\mathrm{c}}=H_{\mathrm{a}, \mathrm{g}}^{\mathrm{c}}=\lambda / D \cdot d \cdot(\operatorname{Re} \cdot \operatorname{Pr} \cdot D / L)^{e}
$$

W. M. Kays [13] donne les valeurs de 1,75 pour $d$ et de 0,33 pour $e$ dans le cas de l'air.

En ce qui concerne les termes de rayonnement, nous avons linéarisé localement le coefficient d'échange radiatif qui est recalculé à chaque pas de temps.

Remarque: Les échanges schématisés sur la figure 2 sont en $R=1 / H$, avec les indices correspondants.

\subsection{CARACTÉRisATION MATHÉMATIQUE DU} MODÈLE. - Le modèle que nous venons de présenter peut se mettre sous la forme classique suivante :

$$
\begin{gathered}
A \cdot \dot{X}=B(X, U) \cdot X+C(X, U) \cdot U \\
Y=D(X, U) \cdot X
\end{gathered}
$$

avec

$$
\begin{array}{ll}
X & : \text { vecteur d'état du modèle } \\
Y & : \text { grandeurs de sortie } \\
U & : \text { grandeurs d'entrée }
\end{array}
$$

$A, B, C, D:$ matrices. Les trois dernières sont fonction des entrées et des états.

Sur un pas de temps d'une minute, les entrées sont maintenues constantes par le système de pilotage. L'état du capteur variant peu, les coefficients sont linéarisés, ce qui permet de résoudre explicitement le système :

$$
\begin{aligned}
& X_{j}=B^{\prime}\left(X_{j-1}, U_{j}\right) \cdot X_{j-1}+C^{\prime}\left(X_{j-1}, U_{j}\right) \cdot U_{j} \\
& X_{j}=D^{\prime}\left(X_{j}, U_{j}\right) \cdot X_{j} .
\end{aligned}
$$

Si la variation des éléments des matrices $B^{\prime}$, $C^{\prime}$ et $D^{\prime}$ en fonction de l'état $X$ du capteur est assez faible $(\approx 20 \%)$ durant nos expériences, elle dépend par contre fortement des entrées $U(\approx 100 \%)$ : le modèle n'est pas linéaire.

4.1 CRITÈre À APPLIQUeR: IMPORTANCE DES ERREURS DE MESURE. - Le vecteur $|\omega\rangle$ des paramètres à identifier (de dimension 10 dans notre exemple) sera déterminé à partir du vecteur $|\mu\rangle$ des mesures instantanées de la puissance extraite (environ 2500 événements de mesure dans notre exemple, à la cadence d'un événement par minute).

Le modèle, convenablement initialisé (vecteur $\left|\omega_{0}\right\rangle$ des paramètres), permet de calculer à chaque instant la puissance extraite du capteur en fonction des grandeurs d'entrée : on construit ainsi le vecteur $|M\rangle$ des puissances extraites calculées, qui est à comparer au vecteur $|\boldsymbol{\mu}\rangle$.

Pour ajuster les paramètres, nous avons choisi de minimiser l'écart quadratique :

$$
Q^{2}=\left\langle M-\mu\left|V^{-1}\right| M-\mu\right\rangle
$$

avec

$|\mu\rangle:$ vecteur des $N$ mesures

$|M\rangle$ : vecteur des $N$ valeurs calculées correspondantes

$|V|$ : matrice de covariance des $N$ mesures.

Si on suppose les erreurs de mesures non corrélées (ce que nous avons vérifié dans un premier temps), l'expression précédente se réduit à :

$$
Q^{2}=\sum_{j=1}^{N} q_{j}^{2}
$$

avec

$$
q_{j}=\left(M_{j}-\mu_{j}\right) / \varepsilon_{j}
$$

$\mu_{j}$ : puissance extraite mesurée à l'instant $j$

$M_{j}:$ puissance extraite calculée à l'instant $j$

$\varepsilon_{j}$ : propagation des erreurs de mesure sur la différence puissance extraite mesurée-calculée, à l'instant $j$

$N$ : nombre d'événements de mesure.

On voit ainsi que l'écart $Q^{2}$ représente le carré d'une distance mesure-calcul dans un espace de dimension $N$ avec des unités données sur chaque axe par les erreurs de mesure. Il est en effet logique, et indispensable pour une analyse pertinente des données [6], que les points des mesures imposent sur le modèle une contrainte d'autant plus grande que leur incertitude est faible.

Lorsque l'écart type des erreurs de mesure des différentes grandeurs n'est pas constant ou que le modèle est non linéaire, l'erreur $\varepsilon_{j}$ doit être estimée à chaque instant $j$. Tel est notre cas et nous présentons ces calculs en annexe.

Lorsque les erreurs de mesures sont gaussiennes, indépendantes, et que le modèle est linéaire, on dispose d'une théorie complète sur cette méthode de minimisation $[5,6,14]$ : la variable aléatoire $Q^{2}$ a la

critère d'ajustement est souvent appelé minimisation de $\chi^{2}$ ) et les propriétés statistiques de cette distribution permettent d'analyser la cohérence de la procédure d'identification d'une part, d'interpréter rigoureusement les résultats d'autre part.

Ces hypothèses sont rarement vérifiées en ce qui concerne les systèmes réels, et notre capteur ne fait pas exception à cette règle. La robustesse de la méthode de minimisation du $\chi^{2}$ permet néanmoins son utilisation de façon assez générale $[5,6,14]$. Cependant, pour pouvoir utiliser certaines proprié- 
tés, nous avons vérifié par tirage de Monte Carlo que la distribution théorique de $q_{j}$ est quasi normale (cf. paragraphe 7.1.2).

4.2 Minimisation DE L'ÉCART QUADRATIQUe; INCERTITUDE SUR LES PARAMÈTRES. - Pour la minimisation proprement dite, nous avons utilisé le programme «MINUIT » développé par le CERN [15], qui permet d'ajuster jusqu'à 55 paramètres libres. A partir des valeurs initiales, le programme utilise d'abord un simplex pour calculer une nouvelle valeur des paramètres libres. Puisque lorsque l'on est suffisamment proche d'un minimum, ce programme utilise un algorithme de type gradient conjugué, avec une métrique particulière (Variable Metric Method) [16]. Il est également possible de calculer les coefficients de corrélations entre paramètres ainsi que les incertitudes sur la valeur des paramètres à partir de la matrice de covariance, et d'effectuer différentes analyses de sensibilité paramétrique près du minimum.
Pour appliquer la méthode des gradients conjugués, on part d'une valeur initiale $\left|\omega_{0}\right\rangle$ des paramètres et on cherche la valeur $\left|\omega_{1}\right\rangle$ pour laquelle $\partial Q^{2} / \partial \omega$ s'annule, en développant au premier ordre cette dérivée. On obtient :

$$
|\mathrm{d} \omega\rangle=-\left(\overline{\overline{\partial^{2} Q^{2}}} \frac{\partial \omega^{2}}{\partial \omega_{0}} \cdot\left|\frac{\partial Q^{2}}{\partial \omega}\right\rangle_{\omega_{0}}\right.
$$

avec

$$
|\mathrm{d} \omega\rangle=\left|\omega_{1}\right\rangle-\left|\omega_{0}\right\rangle .
$$

Lorsque le modèle est linéaire, on obtient directement la valeur des paramètres; sinon, il faut itérer.

La matrice des dérivées secondes de l'écart $Q^{2}$ s'écrit :

$$
\begin{aligned}
& \left(\overline{\overline{\frac{\partial^{2} Q^{2}}{\partial \omega^{2}}}}\right)=2\left(\overline{\overline{\frac{\partial^{2} M}{\partial \omega^{2}}}}\right)^{T} \cdot V^{-1} \cdot|M-\mu\rangle+ \\
& +2\left(\frac{\overline{\overline{\partial M}}}{\partial \omega}\right)^{T} \cdot V^{-1} \cdot\left(\frac{\overline{\overline{\partial M}}}{\partial \omega}\right) \text {. }
\end{aligned}
$$

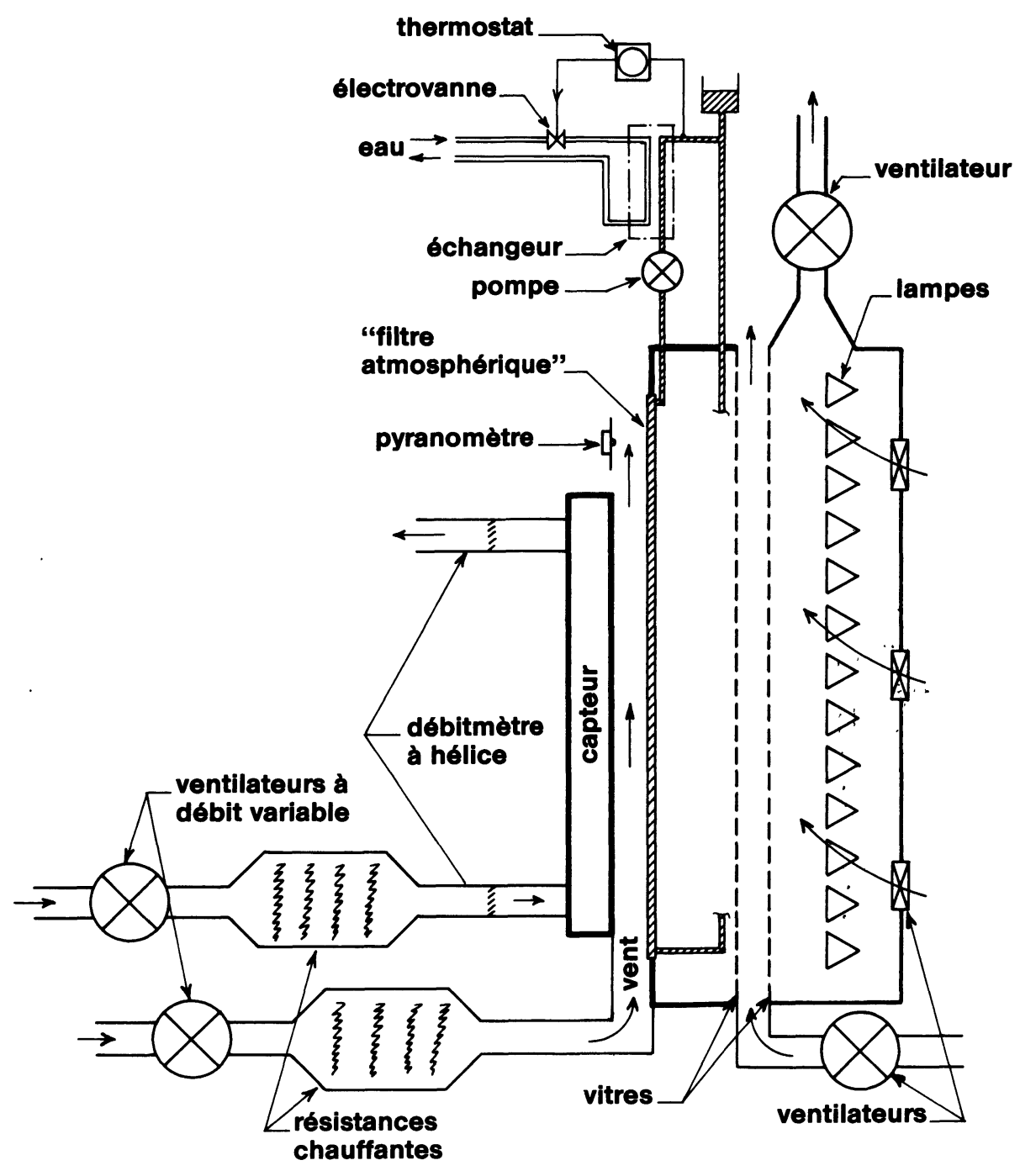

Fig. 4. - Coupe schématique du banc d'essai.

[Schematic cross-section of the testing bench.] 


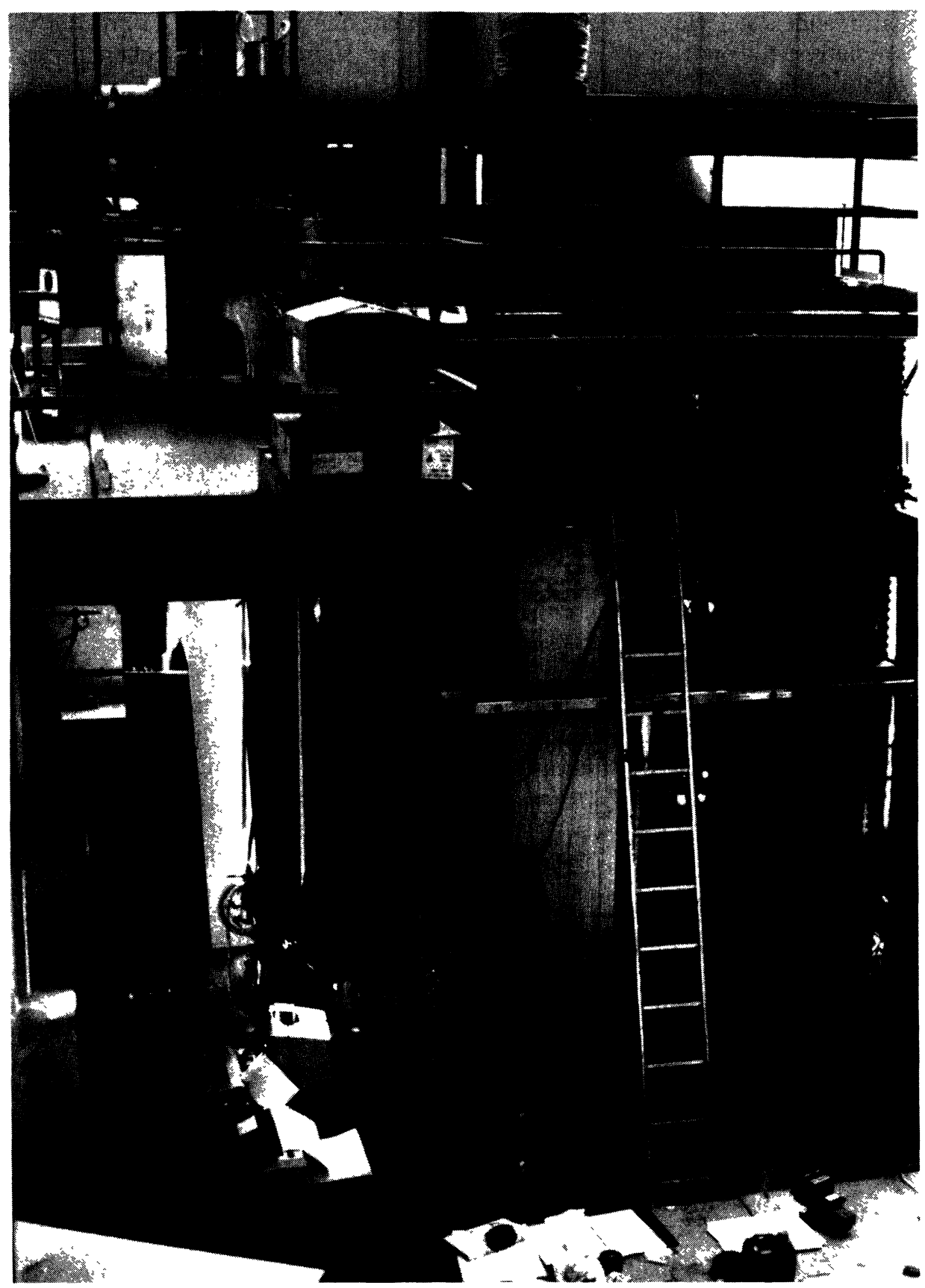

Fig. 5. - Vue générale de l'insolateur.

[General view of the solar simulator.]

Si la sortie du modèle est une fonction linéaire de chacun des paramètres, cette expression se simplifie :

$\left(\frac{\overline{\overline{\partial^{2} Q^{2}}}}{\partial \omega^{2}}\right)=2\left(\overline{\overline{\frac{\partial M}{\partial \omega}}}\right)^{T} \cdot V^{-1} \cdot\left(\overline{\overline{\frac{\partial M}{\partial \omega}}}\right)=2 \cdot A$

$A$ étant alors une forme quadratique.

L'inverse $A^{-1}$ de cette matrice correspond, pour des modèles linéaires, à la matrice de covariance (ou « d'erreur ») paramétrique $[5,6,16]$.
On remplace dans l'équation (8) la dérivée première de $Q^{2}$ par son expression et on obtient :

$$
|\mathrm{d} \omega\rangle=-A^{-1} \cdot\left(\frac{\overline{\overline{\partial M}}}{\partial \omega}\right)^{T} \cdot V^{-1} \cdot|M-\mu\rangle .
$$

On en déduit :

$$
\left(\overline{\overline{\frac{\partial \omega}{\partial \mu}}}\right)=A^{-1} \cdot\left(\overline{\overline{\frac{\partial M}{\partial \omega}}}\right)^{T} \cdot V^{-1} .
$$


On projette maintenant les erreurs de mesures sur la valeur des paramètres. La matrice de covariance des erreurs de mesure s'écrit :

$$
V=|\delta \mu\rangle\langle\delta \mu| .
$$

On pose la matrice de covariance paramétrique :

$\Pi=|\delta \omega\rangle\left\langle\delta \omega\left|=\left(\frac{\overline{\overline{\partial \omega}}}{\partial \mu}\right)\right| \delta \mu\right\rangle\langle\delta \mu|\left(\frac{\overline{\overline{\partial \omega}}}{\partial \mu}\right)^{T}$.

Les matrices $A$ et $V$ étant symétriques, on utilise les équations (10), (12) et (13) pour simplifier l'expression (14). On obtient alors :

$$
\Pi=A^{-1} \text {. }
$$

La matrice des dérivées secondes $A$ nous permet ainsi d'avoir accès à la matrice de covariance paramétrique et donc d'estimer les coefficients de corrélation entre paramètres ainsi que l'incertitude sur la valeur de chacun des paramètres provenant des erreurs de mesure. Le calcul des matrices dérivées est effectué numériquement par le programme MINUIT à partir des valeurs de $Q^{2}$ fournis au minimum.

\section{Expérimentation.}

5.1 Présentation Du banC D'ESSAI. - Dans le cadre d'une procédure d'identification, telle que nous l'avons choisie, le rôle déterminant du banc d'essai est de piloter les grandeurs dynamiques d'entrée pour découpler au mieux les paramètres du modèle tout en restant dans un domaine de fonction- nement quasi réel. Afin de préciser ceci, nous avons - par simulation - recherché les expériences à réaliser et en avons déduit un cahier des charges du banc d'essai, comprenant la plage et la dynamique de variation des grandeurs d'entrée ainsi que la précision requise pour l'ensemble des mesures.

L'insolateur que nous avons réalisé peut être schématisé de la façon suivante (Figs. 4 et 5): un mur de $12 \mathrm{~m}^{2}$, composé de 429 lampes à filament de tungstène de $150 \mathrm{~W}$ ventilées, permet de créer 17 niveaux d'éclairement de 130 à $1150 \mathrm{~W} / \mathrm{m}^{2}$ sur une surface verticale de $6 \mathrm{~m}^{2}$, sans modification du spectre et en conservant une bonne homogénéité spatiale de l'éclairement.

Un "filtre atmosphérique ", dont on pilote la température, permet de contrôler les échanges radiatifs grandes longueurs d'onde avec le capteur. Les échanges convectifs avec l'environnement sont également contrôlés, par pilotage de la température et de la vitesse de l'air en face avant du capteur. Pouı le capteur lui-même sont commandés le débit et la température d'entrée de l'air.

L'ensemble des mesures et des signaux de commande est géré par un système d'acquisition automatique que nous avons entièrement réalisé.

Les publications [2] et [3] contiennent de plus amples précisions sur la conception, la réalisation et les caractéristiques de ce banc d'essai.

5.2 ANAlyse des erreurs de Mesure. - Pour le critère d'identification choisi, nous avons déjà mentionné l'importance de la détermination des incertitudes expérimentales. De plus, cette information est nécessaire pour évaluer la précision du modèle produit.

Tableau I. - Plage et dynamique de variation des grandeurs physiques d'entrée et précision de mesure du dispositif expérimental réalisé.

\begin{tabular}{|c|c|c|c|c|c|}
\hline Grandeur & Plage de variation & $\begin{array}{l}\text { Pas élémentaire } \\
\text { du pilotage }\end{array}$ & Temps de réponse & $\begin{array}{l}\text { Homogénéité } \\
\text { spatiale }\end{array}$ & $\begin{array}{c}\text { Erreur de } \\
\text { mesure }\end{array}$ \\
\hline $\begin{array}{l}\text { Eclairement } \\
\text { Débit d'air } \\
\text { dans le cap. }\end{array}$ & $\begin{array}{c}130 \text { à } 1150 \mathrm{~W} / \mathrm{m}^{2} \\
0 \text { à } 260 \mathrm{~m}^{3} / \mathrm{h}\end{array}$ & $\begin{array}{l}65 \mathrm{~W} / \mathrm{m}^{2} \\
1 \mathrm{~m}^{3} / \mathrm{h}\end{array}$ & $\begin{array}{l}\text { qq secondes } \\
\text { qq secondes }\end{array}$ & $\begin{array}{c}6 \% \\
-\end{array}$ & $\begin{array}{l}1,3 \% \\
0,5 \%\end{array}$ \\
\hline $\begin{array}{l}\text { Fuite d'air } \\
\text { dans le cap. }\end{array}$ & - & - & - & - & $0,5 \%$ \\
\hline $\begin{array}{l}\text { Temp. d'entrée } \\
\text { dans le cap. }\end{array}$ & 20 à $80^{\circ}$ & $1^{\circ}$ & 5 minutes & - & $0,04^{\circ}$ \\
\hline $\begin{array}{l}\text { Dif. de temp. } \\
\text { entrée-sortie }\end{array}$ & - & - & - & - & $0,06^{\circ}$ \\
\hline Temp. de ciel & 15 à $40^{\circ}$ & $1^{\circ}$ & 5 minutes & $1,5^{\circ}$ & $0,2^{\circ}$ \\
\hline $\begin{array}{l}\text { Temp. de l'air } \\
\text { devant le cap. }\end{array}$ & 20 à $40^{\circ}$ & $5^{\circ}$ & 2 minutes & $0,5^{\circ}$ & $0,5^{\circ}$ \\
\hline $\begin{array}{l}\text { Vitesse de } \\
\text { l'air (vent) }\end{array}$ & 0 à $2 \mathrm{~m} / \mathrm{s}$ & continu & qq secondes & $0,2 \mathrm{~m} / \mathrm{s}$ & $0,1 \mathrm{~m} / \mathrm{s}$ \\
\hline
\end{tabular}

[Precision, range and time variation of input quantities of the test facilities.] 
Pour estimer les erreurs instrumentales dans les conditions expérimentales réelles, nous avons mesuré de façon redondante et pendant toute la durée des expériences chaque grandeur physique, puis effectué une analyse statistique fine $[2,3]$. L'ensemble de ces résultats est résumé dans le tableau I, dans lequel les erreurs dépendant du débit sont calculées pour une valeur de débit de $60 \mathrm{~m}^{3} / \mathrm{h}$. Ces erreurs ont été ensuite propagées sur la différence puissance extraite mesurée-calculée en suivant la méthode présentée en annexe.

Un cas particulier est celui de l'état initial du capteur (début d'une séquence expérimentale), qui est mal connu en raison de l'absence de mesures directes à l'intérieur du capteur. Cette erreur a été estimée en fonction des fluctuations de la température du hall avant chaque expérience, puis propagée par le modèle. L'effet de cette erreur ne devient négligeable, aux faibles débits, qu'après une heure de fonctionnement du capteur [2].

5.3 Plan D'EXPÉRIENCE. - Dans un premier temps, nous avons conçu trois types d'expériences afin de découpler phénoménologiquement les paramètres du modèle : pour le premier type, on souffle de l'air chaud dans le capteur dont on a isolé thermiquement la face avant de l'extérieur; la circulation de l'air est alors inversée pour conserver le sens des gradients thermiques. Le deuxième type d'expériences est similaire, mais sans isolation devant le capteur. On fait alors varier indépendamment les grandeurs physiques intervenant dans les échanges avant: température et vitesse de l'air devant le capteur, température de "ciel». Pour le troisième type, on souffle de l'air normalement dans le capteur et on génère des séquences d'éclairement, soit purement artificielles, soit correspondant à des journées réelles.

Une deuxième étape consiste à favoriser un découplage dynamique; pour chaque expérience, nous avons recherché par simulation la dynamique de variation des grandeurs d'entrée qui différencient au mieux les paramètres libres du modèle [2].

Nous nommerons les trois catégories d'expériences que nous venons de présenter " $A$ » (débit inversé et face avant isolée), "B » (soufflage inversé d'air

expériences ont été réalisées en maintenant le débit d'air constant pour chacune d'elles, à des valeurs correspondant à la plage de fonctionnement pour lesquelles le capteur a été conçu : de 50 à $240 \mathrm{~m}^{3} / \mathrm{h}$. L'écoulement est alors turbulent.

Deux autres types d'expériences ont également été réalisées. Pour les expériences « $\mathrm{D}$ », le débit d'air est faible (inférieur à $50 \mathrm{~m}^{3} / \mathrm{h}$ ) tandis que pour les expériences « $\mathrm{E}$ », soit la dynamique est particulièrement importante, soit le débit n'est pas constant : alors que l'éclairement fluctue, on commande le débit de façon à maintenir constante la température de sortie d'air du capteur.

Seules les expériences « $A$ », « $B$ » et « $C$ » seront utilisées pour l'identification des paramètres. Les expériences « $D$ » et « $E$ » permettront de tester le domaine de validité du modèle.

\section{Ajustement des paramètres.}

L'ajustement des paramètres n'est pas un processus séquentiel, mais au contraire fortement bouclé : les résultats obtenus à un moment donné peuvent remettre en cause les étapes précédentes. Dans les paragraphes suivants, nous avons tenté de synthétiser la démarche de façon linéaire sans toutefois masquer les difficultés rencontrées et les retours en arrière.

6.1 ChoiX DeS PARAMÈTRes libres. - Le choix des paramètres libres est une étape importante de l'identification qui met en œuvre une expertise physique. Un nombre trop élevé de paramètres libres conduit, outre les singularités numériques inhérentes, à des paramètres trop corrélés entre eux : le minimum obtenu est alors très plat et la valeur finale des paramètres perd sa signification physique. Lorsque le nombre de paramètres libres est au contraire trop faible, un effet inverse conduit également à dépourvoir les paramètres de leur sens physique prévu : certains paramètres ont à rendre compte de plusieurs phénomènes à la fois, ce qui fait dégénérer le modèle vers un modèle boîte noire...

Lors de la mise en œuvre de la procédure d'identification, le nombre de paramètres libres a évolué en fonction du modèle et des expériences utilisés ainsi qu'en fonction de l'intelligibilité des résultats. Nous présentons ici ce qui correspond à la phase finale de notre travail (pour les autres phases, voir [2]).

Les paramètres physiques du modèle décrit précédemment sont au nombre d'une trentaine. Notre objectif étant de simuler avec précision la puissance extraite du capteur, de comprendre les phénomènes physiques intervenant de façon prépondérante sur cette puissance et non de déterminer avec précision l'ensemble des paramètres macrophysiques du capteur, un paramètre du modèle sera ajusté (sera libre) si :

- l'incertitude a priori de ce paramètre a un effet non négligeable sur la précision souhaitée du modèle ;

- la sensibilité de la puissance extraite par rapport à ce paramètre est non totalement corrélée à la sensibilité de l'ensemble des autres paramètres libres (détermination d'une base irréductible de paramètres).

Des résultats de simulation préliminaire nous ont permis de trouver une douzaine de paramètres ayant une influence non négligeable sur la puissance 
Tableau II. - Sensibilité moyenne de la puissance extraite aux principaux paramètres du modèle, et ce pour les trois types d'expériences $\mathrm{A}, \mathrm{B}$ et $\mathrm{C}$ (cf. Eq. (16)).

[Average extracted heat sensitivity to the main model parameters, for the three classes of experimental sequences A, B and C (cf. Eq. (16).]

\begin{tabular}{|c|c|c|c|c|c|c|c|c|c|c|c|c|}
\hline $\begin{array}{c}\text { Type } \\
\text { d'expérience }\end{array}$ & $\alpha_{\mathrm{a}}$ & $\begin{array}{c}H_{\mathrm{e}, \mathrm{v}}^{\mathrm{c}} \\
{\left[\mathrm{W} / \mathrm{m}^{2} \mathrm{~K}\right]}\end{array}$ & $\begin{array}{l}b \\
{[H}\end{array}$ & {$\left[\begin{array}{c}c \\
a, f\end{array}\right]$} & $\begin{array}{c}C_{\mathrm{i}} \\
{\left[\mathrm{J} / \mathrm{m}^{2} \mathrm{~K}\right]}\end{array}$ & $\begin{array}{c}C_{\mathrm{p}} \\
{\left[\mathrm{J} / \mathrm{m}^{2} \mathrm{~K}\right]}\end{array}$ & $\begin{array}{c}H_{\mathrm{i}} \\
{\left[\mathrm{W} / \mathrm{m}^{2} \mathrm{~K}\right]}\end{array}$ & $\alpha_{\mathrm{v}}$ & $\begin{array}{c}d \\
{\left[H_{\mathrm{a}, \mathrm{v}}^{\mathrm{c}}\right]}\end{array}$ & $\begin{array}{c}H_{\mathrm{ar}} \\
{\left[\mathrm{W} / \mathrm{m}^{2} \mathrm{~K}\right]}\end{array}$ & $\underset{\left[\mathrm{J} / \mathrm{m}^{2} \mathrm{~K}\right]}{C_{\mathrm{vt}}}$ & $\begin{array}{c}H_{\mathrm{v}, \mathrm{c}}^{\mathrm{r}} \\
{\left[\mathrm{W} / \mathrm{m}^{2} \mathrm{~K}\right]}\end{array}$ \\
\hline «A $»$ & - & - & $17 \%$ & $30 \%$ & $9 \%$ & $8 \%$ & $8 \%$ & - & $25 \%$ & $40 \%$ & $6 \%$ & - \\
\hline 《B » & - & $10 \%$ & $25 \%$ & $80 \%$ & $11 \%$ & $10 \%$ & $10 \%$ & - & $15 \%$ & - & $45 \%$ & $12 \%$ \\
\hline «C $\rangle$ & $0,3 \%$ & $22 \%$ & $20 \%$ & $60 \%$ & $40 \%$ & $40 \%$ & $50 \%$ & $\begin{array}{c}20 \% \\
(0,15 \pm 0,03)\end{array}$ & $100 \%$ & - & $50 \%$ & $45 \%$ \\
\hline
\end{tabular}

thermique. Dans le tableau II nous avons porté pour chacun d'eux la variation $\delta \omega_{n}$ qui entraîne une modification de la puissance thermique modélisée égale à l'erreur de mesure, c'est-à-dire :

$$
\frac{1}{N} \sum_{j=1}^{N} \frac{1}{\varepsilon_{j}}\left|\frac{\partial M_{j}}{\partial \omega_{n}}\right| \delta \omega_{n}=1 .
$$

Ces douze paramètres n'ont pas été maintenus libres lors du processus d'identification, certains d'entre eux étant trop corrélés aux autres. Ainsi la capacité thermique de la vitre $\left(C_{\mathrm{v}}\right)$ n'a pas été ajustée et quatre autres paramètres ont été ajustés empiriquement : $\alpha_{\mathrm{v}}, H_{\mathrm{v}, \mathrm{e}}^{\mathrm{c}}, H_{\mathrm{ar}}, H_{\mathrm{c}, \mathrm{v}}^{\mathrm{r}}$. Les 7 paramètres restants ont été laissés libres dans la phase finale de l'ajustement.

6.2 VALEUR INITIALE DES PARAMÈTRES; AJUSTEMENT EMPIRIQUE. - Lorsque les valeurs initiales des paramètres sont trop éloignées de leur valeur au minimum absolu, les risques de tomber dans des minima secondaires lors de l'ajustement par minimisation sont nombreux. S'il existe quelques outils pour éprouver la qualité du minimum (exploration du voisinage par tirage de Monte Carlo, étude de sensibilité paramétrique, analyse physique de la valeur des paramètres, modification des valeurs initiales...), il est de toute façon illusoire d'espérer atteindre le minimum absolu en injectant des valeurs initiales quelconques dans un modèle fortement non linéaire. Ainsi, la phase d'ajustement préliminaire (ou empirique) est déterminante ; elle requiert tous les moyens classiques de l'heuristique physique.

Dans un premier temps, nous avons calculé les valeurs de paramètres ainsi que leurs limites (Tab. III) à partir des propriétés thermophysiques des matériaux, de la géométrie du capteur et d'une étude expérimentale préliminaire [17].

Dans un deuxième temps, nous avons ajusté empiriquement les quelques paramètres intervenant sur le comportement statique du capteur. Pour cela, nous nous sommes basés sur les considérations physiques simples, telle la conservation des puissances intégrées sur certaines périodes expérimentales.

Pour les expériences « $\mathrm{A}$ », qui sont les plus simples, nous avons obtenu des biais mesure-calcul de 30 à $50 \%$ sur la puissance intégrée sur chaque expérience. Des analyses de sensibilité ont montré que seule une estimation trop faible de la conductance du fond pouvait expliquer ce biais ; nous avons pu ainsi estimer une nouvelle valeur de cette conductance.

Pour les expériences « $\mathrm{B}$ », les pertes thermiques calculées se sont aussi révélées insuffisantes, ce qui nous a conduit à augmenter de façon considérable les échanges entre la vitre et l'absorbeur, et donc à

Tableau III. - Tableau récapitulatif schématisant l'évolution de la valeur des 10 paramètres libres au cours de l'identification.

[Table summarizing the evolution of the 10 parameters value along with the identification process.]

\begin{tabular}{|c|c|c|c|c|c|c|c|c|}
\hline & $\alpha_{\mathrm{a}}$ & $\begin{array}{c}H_{\mathrm{e}, \mathrm{v}}^{\mathrm{c}} \\
{\left[\mathrm{W} / \mathrm{m}^{2} \mathrm{~K}\right]}\end{array}$ & $b$ & $n$ & $\begin{array}{c}C_{\mathrm{i}} \\
{\left[\mathrm{J} / \mathrm{m}^{2} \mathrm{~K}\right]}\end{array}$ & $\underset{\left[\mathrm{J} / \mathrm{m}^{2} \mathrm{~K}\right]}{C_{\mathrm{p}}}$ & $\begin{array}{c}H_{\mathrm{i}} \\
{\left[\mathrm{W} / \mathrm{m}^{2} \mathrm{~K}\right]}\end{array}$ & $Q^{2} / N$ \\
\hline Valeur initiale & 0,85 & 0 & 0,70 & 0,61 & 8000 & 4000 & 10 & 100 \\
\hline $\begin{array}{c}\text { Valeur après ajustement } \\
\text { empirique }\end{array}$ & 0,95 & 9,3 & 0,42 & 1,1 & 11000 & 14000 & 20 & 15 \\
\hline $\begin{array}{l}\text { Valeur après ajustement } \\
\text { par minimisation }\end{array}$ & 0,927 & 7,4 & 0,96 & 0,64 & 6900 & 6800 & 12,1 & 1,1 \\
\hline $\begin{array}{c}\text { Limites des valeurs } \\
\text { attendues }\end{array}$ & $\begin{array}{l}0,75 \\
\text { à } 0,95\end{array}$ & $\begin{array}{l}6 \\
\text { à } 12\end{array}$ & $\begin{array}{l}0,7 \\
\text { à } 1,3\end{array}$ & $\begin{array}{l}0,5 \\
\text { à } 0,8\end{array}$ & $\begin{array}{r}4000 \\
\text { à } 8000\end{array}$ & $\begin{array}{r}2000 \\
\text { à } 4000\end{array}$ & $\begin{array}{c}2 \\
\text { à } 12\end{array}$ & \\
\hline
\end{tabular}




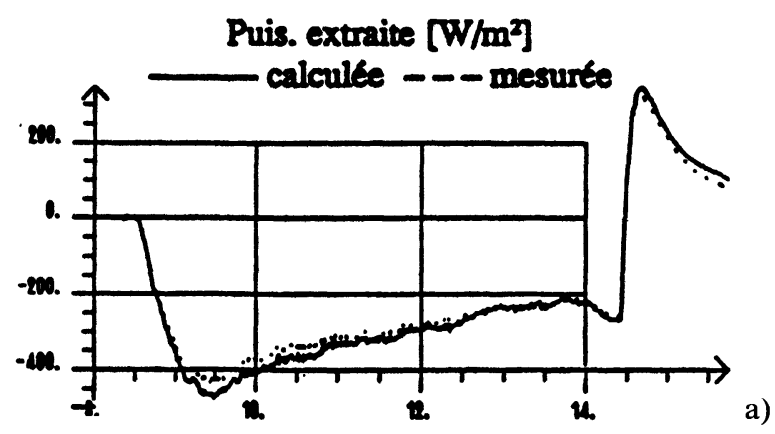

Différence mesure-modèle $\left[\mathrm{W} / \mathrm{m}^{2}\right]$

Temp. de vitre $\left[{ }^{\circ} \mathrm{C}\right]$

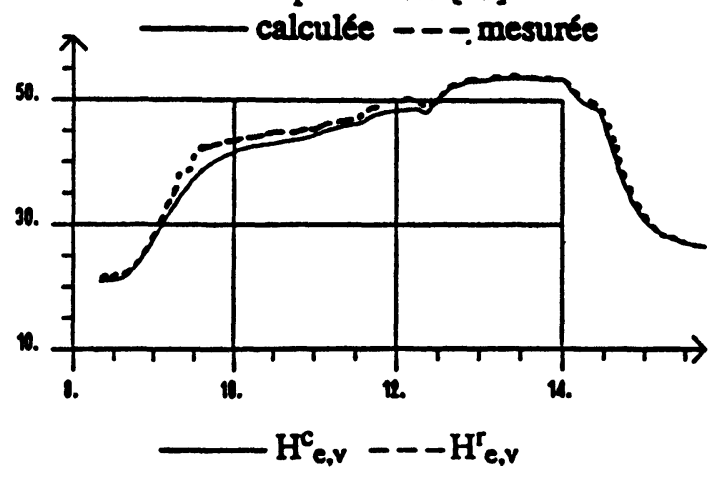

a)
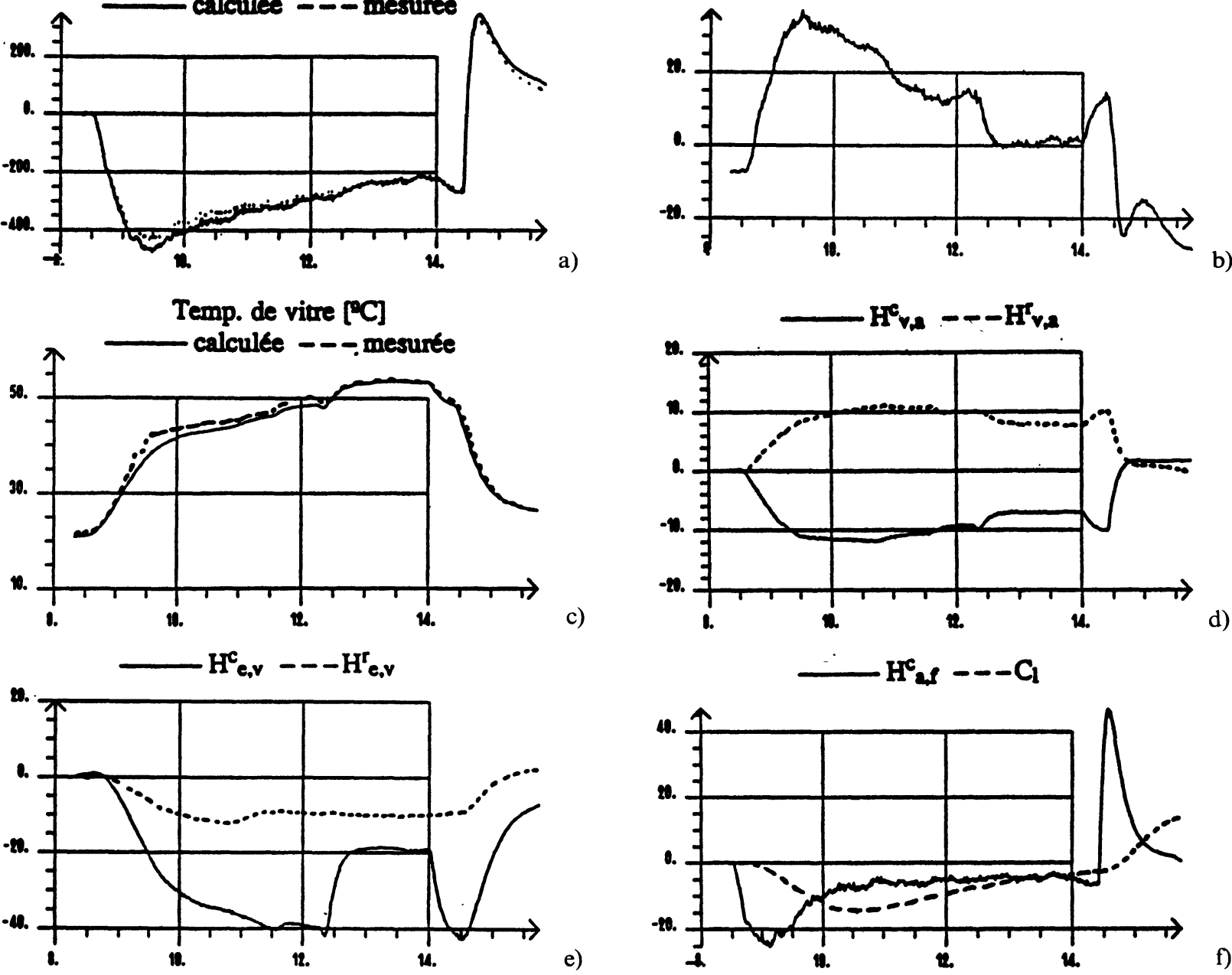

e)

$\mathrm{Hr}^{\mathrm{ap}}$

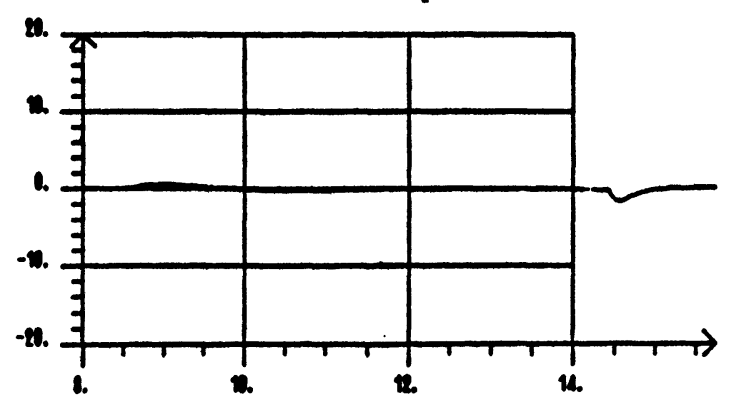

g)

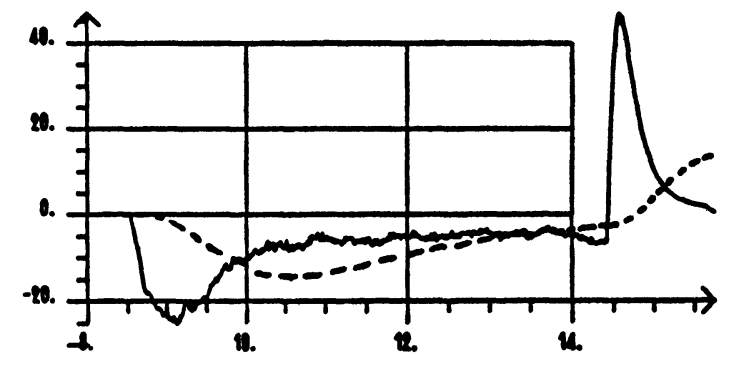

$\mathrm{H}_{1}^{\mathrm{k}_{1}}---\mathrm{C}_{\mathrm{v}}$
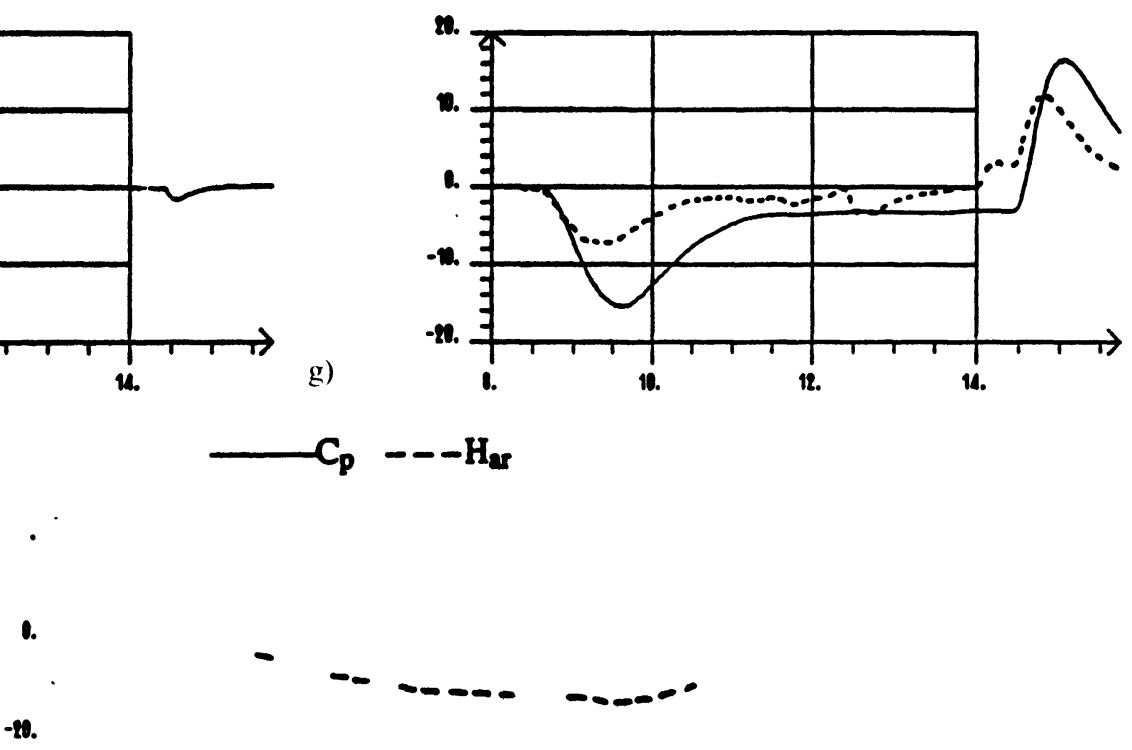

8. u. u. u. i)

Fig. 6. - Tracé, en fonction du temps, de la puissance thermique extraite du capteur (courbe a), de l'écart mesuremodèle de cette puissance (courbe b), de la température de vitre (courbe c) ainsi que de la sensibilité de la puissance thermique en fonction des principaux paramètres $\omega_{n}$ (courbes $d$ à i).

[Time dependance of : a) the extracted heat from collector; b) model-measurement deviation of the extracted heat ;

c) glazing temperature; d) to i) extracted heat sensitivity to the main parameters.] 
supposer l'existence d'une circulation parasite d'air dans l'enceinte avant. Cette hypothèse a été confirmée par la suite, lorsque le démontage du capteur a laissé apparaître une mauvaise liaison entre l'absorbeur et le caisson isolant. La valeur de ce débit parasite d'air a été fixée de façon empirique à $10 \%$ du débit d'entrée [2]. Le modèle prenant en compte cette circulation parasite a été présenté paragraphe 3.2.

Enfin, nous avons ajusté les principaux paramètres intervenant sur la dynamique du modèle. Pour cela, nous avons tracé - en fonction du temps - l'écart mesure-modèle de la puissance extraite et les dérivées $\partial M_{i} / \partial \omega_{n}$ de la puissance calculée en fonction de chaque paramètre $\omega_{n}$. Un exemple d'un tel tracé est représenté figure 6. On peut par exemple s'y convaincre que les variations rapides de l'écart mesure-modèle aux instants $12,5 \mathrm{~h}$ et $14 \mathrm{~h}$ (courbe b) sont dues à une mauvaise valeur du coefficient $H_{\mathrm{e}, \mathrm{v}}^{\mathrm{c}}$ (courbe e). Par contre, de nombreux coefficients peuvent expliquer l'écart mesure-modèle en début d'expérience (entre $9 \mathrm{~h}$ et $11 \mathrm{~h}$ ); d'autres expériences découplant mieux ces paramètres ont été utilisées.

Les capacités calorifiques et les coefficients d'échange paroi-fluide arrière ont ainsi été ajustés. Lorsque l'écart moyen mesure-modèle atteint $10 \%$ environ, le fort couplage entre paramètres ne nous permet pas d'induire de nouvelles valeurs diminuant cet écart ; on passe alors à l'ajustement par minimisation.

La durée et la difficulté de cet ajustement dépendent beaucoup du système étudié : plus son fonctionnement est éloigné de ce qu'on attend (ce qui fut notre cas), plus cette étape est longue et cruciale ; à la limite, on peut être amené à reconcevoir le plan d'expérience et le modèle.

\subsection{AJUSTEMENT PAR MINIMISATION DE $\chi^{2}$.}

6.3.1 Ajustement par étape. - Afin d'estimer la pertinence de la minimisation et la cohérence de la démarche, nous avons d'abord effectué des minimisations restreintes à différentes séquences expérimentales.

Les expériences où l'on souffle de l'air chaud ayant à la fois le plus petit nombre de paramètres libres et pour ceux-ci la plus forte sensibilité, nous avons suivi la progression suivante dans l'identification: les paramètres sont d'abord ajustés sur les expériences « $\mathrm{A}$ » (avec 6 paramètres libres), puis sur les expériences « $\mathrm{A}$ » + « $\mathrm{B}$ » (avec 8 paramètres libres), et enfin sur les expériences « $A$ » « $B »+\langle C$ »(10 paramètres libres).

Lors de la mise en œuvre de ces ajustements, nous avons pu faire les observations suivantes:

- la valeur du coefficient d'échange convectif absorbeur-fluide est aberrante : la valeur de l'expo- sant $\mathrm{n}$ de l'expression de ce coefficient est de 1,1 alors que les lois expérimentales que l'on trouve dans la littérature donnent des valeurs de cet exposant comprises entre 0,5 et 0,9 ;

- toujours pour ce coefficient d'échange, les paramètres $\mathrm{b}$ et $\mathrm{n}$ sont sensiblement différents selon que l'on minimise l'écart sur les expériences $\langle A »+\langle B »$ ou sur $\langle A\rangle+\langle B »+\langle C\rangle$;

- pour les expériences avec éclairement, la concordance mesure-modèle n'est pas satisfaisante.

Cela nous a amené à remettre en cause les expériences avec soufflage renversé d'air chaud face avant isolée ( $\mathrm{A} »)$ : pour ces expériences en effet, la puissance extraite est très sensible au coefficient d'échange convectif absorbeur-fluide lorsque la dynamique est forte, et on peut soupçonner qu'une dynamique trop élevée fasse sortir l'expérience du domaine de validité du modèle. Pour vérifier cette hypothèse, nous avons effectué une minimisation avec coupure de $\chi^{2}$, c'est-à-dire une minimisation pour laquelle tous les événements dont l'écart quadratique pondéré $q_{j}^{2}$ est supérieur à un certain seuil ne sont pas utilisés pour le calcul de $Q^{2}$. Il apparaît alors que tous les points de mesure des expériences « $A$ » pour lesquels la dynamique est forte sont " exclus » de la minimisation $[2,3]$.

Ces expériences ont donc été supprimées dans la suite de l'identification. Les expériences " $A$ » ont été uniquement utilisées pour déterminer la conductance arrière $H_{\text {ar }}$, suffisamment découplée des autres paramètres.

6.3.2 Ajustement global sur les expériences retenues. - Nous avons finalement minimisé l'écart quadratique pondéré $Q^{2}$ sur 7 expériences : 2 « $\mathrm{B} »$ (soufflage d'air chaud sans isolation avant) et 5 « $\mathrm{C}$ » (avec éclairement). Ces expériences totalisent 42 heures d'expériences, avec des mesures toutes les minutes, soit 2500 mesures environ.

Lors de cette phase d'ajustement, la valeur excessive de la conductance de l'isolant du fond nous a conduit à soupçonner l'importance des pertes thermiques dans les caissons de répartition d'air à l'entrée et à la sortie du capteur, qui ont alors été modélisés séparément. D'autre part, la matrice de covariance nous a révélé que certains paramètres libres $\left(C_{\mathrm{v}}\right.$, $\left.\alpha_{\mathrm{v}}, d\right)$ sont totalement corrélés aux autres; ils ont donc été maintenus fixes par la suite.

D'une valeur de $Q^{2}$ par point (c'est-à-dire $Q^{2} / N$ ) supérieure à 100 pour les valeurs $a$ priori des paramètres, cet écart s'est réduit à 15 à la fin de l'ajustement empirique et la minimisation nous a permis d'obtenir la valeur finale de 1,1 (Tab. III) ; les étapes intermédiaires nous ont suggéré des modifications importantes de modélisation afin que les paramètres libres aient une valeur finale pertinente. Nous analysons l'ensemble des résultats dans le paragraphe suivant (paragraphe 7). 
Dans notre cas, et à titre d'exemple, une minimisation avec 7 paramètres libres nécessite typiquement 500 simulations sur les séquences expérimentales utilisées. Cela correspond à la simulation diurne du capteur pendant 5 années, avec un pas de temps de la minute, et nécessite 12 minutes de temps calcul sur l'ordinateur « UNIVAC 1190 » du Centre ParisSud Informatique (1,8 M. Flops), ou 4 heures de calcul sur une station de travail «APOLLO DN $3000 »$.

6.4 REMARQUES CONCERNANT LE PLAN D'EXPÉRIENCE. - Le plan d'expérience initial a été conçu en supposant que l'enceinte avant du capteur était figée. Une fois la circulation parasite d'air mise en évidence, puis modélisée, la matrice de covariance paramétrique a montré une très forte corrélation entre les coefficients d'échange convectif $H_{\mathrm{v}, \mathrm{g}}^{\mathrm{c}}$ et $H_{\mathrm{e}, \mathrm{v}}^{\mathrm{c}}$ : un seul des deux a pu être ajusté. Des expériences avec des échanges avant plus variés en particulier vitesse de "vent " très variable auraient permis de mieux décorréler ces coefficients. Elles n'ont pu être réalisées (banc d'essai cédé à un laboratoire industriel).

Lors de la définition du plan d'expérience, nous avons conçu les séquences « $A$ » (soufflage d'air chaud face avant isolé) afin d'obtenir un découplage phénoménologique important. On s'est ainsi fortement éloigné du fonctionnement ordinaire du système, jusqu'à sortir du domaine de validité du modèle lorsque la dynamique est forte. Cette inadaptation a été mise en évidence par l'ajustement avec coupure de $\chi^{2}$. Pour un nouveau plan d'expériences, si ces expériences étaient retenues, elles ne seraient conduites qu'avec une faible dynamique.

Les expériences « $B$ » relèvent de la même démarche ; on pourrait les remplacer avantageusement par des expériences avec éclairement « $\mathrm{C}$ » pour lesquelles on fait varier les principales grandeurs intervenant dans l'expression des pertes avant (température d'entrée d'air, vitesse et température d'air devant le capteur, température de « ciel »...).

On a ainsi une illustration concrète de ce que nous entendons par la cohérence intrinsèque de la procédure vis-à-vis des objectifs annoncés.

\section{l’identification.}

7.1 ANALYSE STATISTIQUE. - Le choix du critère de minimisation de l'écart quadratique $Q^{2}$, outre sa robustesse, permet d'effectuer différents tests statistiques rigoureux pour les modèles linéaires $[5,6,14]$. Pour les modèles non linéaires - ce qui est notre cas - l'interprétation des résultats n'est pas directe.

7.1.1 Variable aléatoire $Q^{2}$. Pour $N$ points de mesure et pour $K$ paramètres libres, la distribution de la variable $Q^{2}$ est un $\chi^{2}$ à $N-K-1$ degrés de liberté. Son espérance mathématique est $N-K-1$, sa variance $2 \cdot(N-K-1)$. On peut estimer la pertinence du processus d'identification à travers la distribution de $\chi^{2}$ lorsque l'on dispose de nombreuses séquences expérimentales : par exemple, lorsqu'un même capteur a été expérimenté dans différents laboratoires, chacun d'eux ayant ensuite effectué un ajustement. Dans le cas contraire, lorsqu'on réalise une seule identification - ce qui correspond à l'étude présentée - ce test se réduit à juger l'ajustement acceptable si la valeur du $Q^{2}$ réduit (c'est-à-dire $\left.Q^{2} /(N-K-1)\right)$ est inférieure à 1,5 [14]. Dans le cas traité ici, nous obtenons une valeur de 1,1 .

Une valeur trop élevée de $Q^{2}$ eut signifié soit une insuffisance du modèle par rapport à la précision de mesure, soit une mauvaise détermination des erreurs de mesure.

7.1.2 Valeur moyenne et distribution de l'écart $q_{j}$. - La minimisation de $\chi^{2}$ est asymptotiquement non biaisée [5] ; nous avons bien vérifié que la valeur moyenne de $q_{j}$ est nulle aux erreurs de calcul près $(-0,003)$.

De plus, si le système est linéaire, si les erreurs sont gaussiennes et correctement estimées, la distribution théorique de $q_{j}$ suit une loi normale, centrée. Sur la figure 7 nous avons reporé la distribution réelle de $q_{j}$; la différence par rapport à la loi normale peut s'expliquer à la fois par la non-linéarité du modèle et par la non-normalité des erreurs de mesure (notamment pour l'éclairement).

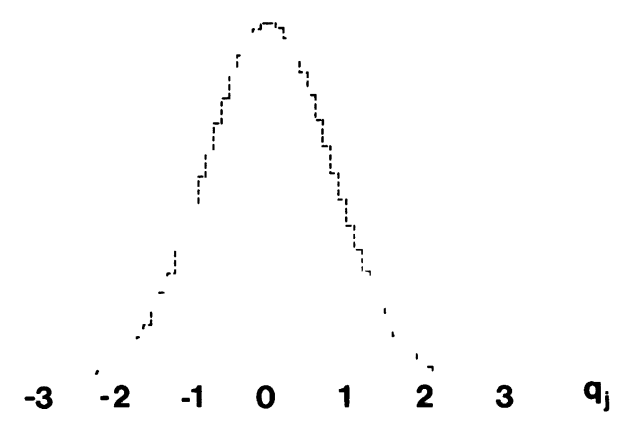

Fig. 7. - ( - Distribution observée de l'écart $q_{j}$. (- - -) Loi normale correspondante.

$\left[(-)\right.$ Experimental distribution of the $q_{j}$ deviation. (- - -) Associated normal law.] 
Pour séparer l'effet de la non-linéarité, nous avons effectué un tirage de Monte Carlo pour des erreurs de mesure supposées gaussiennes; on obtient pour $q_{j}$ la distribution représentée figure 8 , qui est proche d'une loi normale avec une accumulation autour de zéro. Les écarts résiduels entre ces deux distributions (Figs. 7 et 8) sont donc attribuables à la nonnormalité des erreurs de mesure.

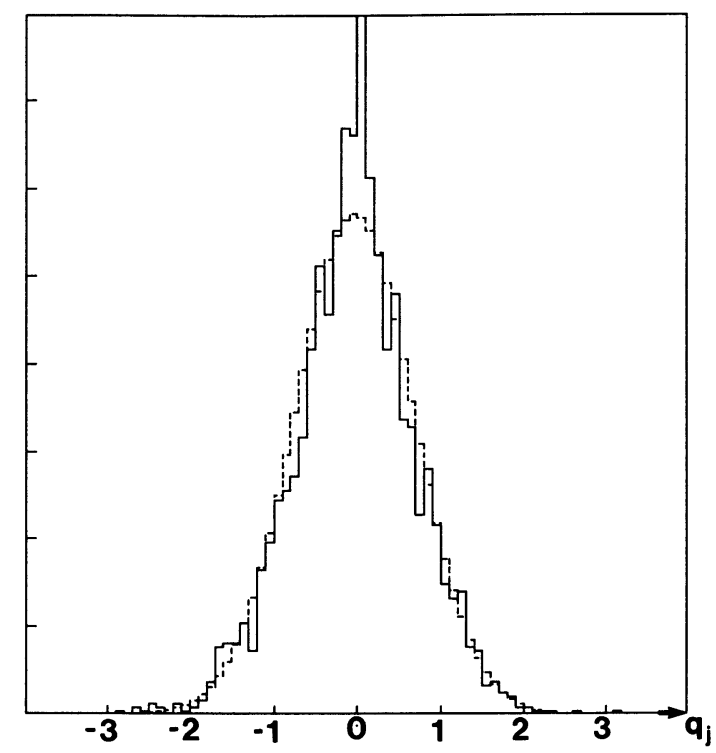

Fig. 8. - (-) Distribution de l'écart $q_{j}$ déterminé par tirage de Monte-Carlo des erreurs de mesure. (- - - ) Loi normale correspondante.

[(- Distribution of the $q_{j}$ deviation, obtained from a Monte-Carlo drawing on the measurements errors. (- - - -) Associated normal law.]

7.1.3 Corrélation et incertitude paramétrique. Pour des modèles linéaires, nous avons vu (paragraphe 4.2) que la méthode des gradients conjugués nous permet de calculer la matrice de covariance des paramètres à partir de la matrice des dérivées secondes de $Q^{2}$, puis d'en, déduire les coefficients de corrélation et les incertitudes statistiques sur les différents paramètres libres.

Cette matrice de covariance paramétrique a été calculée lors de chaque processus d'identification, ce qui a permis de l'utiliser pour le choix des paramètres libres.

Dans le tableau IV nous donnons les valeurs obtenues au minimum pour 7 paramètres libres. La très forte corrélation entre paramètres (sur 21 coefficients, 12 ont une valeur absolue supérieure à 0,5 ) montre que le large éventail d'expériences réalisées ne suffit pas à découpler l'ensemble des paramètres ; d'où les difficultés et les limites de l'ajustement empirique d'une part, l'importance d'une analyse critique des résultats de la minimisation d'autre part.

Tableau IV. - Coefficients de corrélation entre les 7 paramètres libres du modèle.

[Correlation coefficients between the 7 free parameters of the model.]

\begin{tabular}{|r|r|r|c|c|c|c|}
\cline { 2 - 7 } \multicolumn{1}{c|}{} & \multicolumn{1}{c|}{$\alpha_{\mathrm{a}}$} & $H_{\mathrm{e}, \mathrm{v}}^{\mathrm{c}}$ & \multicolumn{1}{c|}{$b$} & $n$ & $C_{\mathrm{i}}$ & $C_{\mathrm{p}}$ \\
\hline$H_{\mathrm{e}, \mathrm{v}}^{\mathrm{c}}$ & 0,80 & & & & & \\
\hline $\begin{array}{r}b \\
{\left[H_{\mathrm{a}, \mathrm{f}}^{\mathrm{c}}\right]}\end{array}$ & $-0,95$ & $-0,65$ & & & & \\
\hline$n$ & $-0,86$ & $-0,68$ & 0,84 & & & \\
\hline$C_{\mathrm{i}}$ & 0,65 & 0,76 & $-0,57$ & $-0,53$ & & \\
\hline$C_{\mathrm{p}}$ & $-0,39$ & $-0,41$ & 0,39 & 0,35 & $-0,68$ & \\
\hline$H_{\mathrm{i}}$ & $-0,22$ & $-0,63$ & 0,01 & 0,19 & $-0,40$ & $-0,16$ \\
\hline
\end{tabular}

La matrice de covariance permet également toujours pour un système linéaire - de déterminer l'incertitude sur les paramètres dus aux erreurs de mesure. Nous donnons ces valeurs pour le cas étudié dans le tableau V. Nous donnons également l'intervalle d'incertitude calculée explicitement de la façon

Tableau V. - Erreur théorique (calculée à partir de la matrice de covariance paramétrique) et erreur calculée explicitement pour les 7 paramètres libres.

[Parametric covariance matrix determination of the error on the 7 free parameters and their explicitly determined values.]

\begin{tabular}{|c|c|c|c|c|c|c|c|}
\hline Paramètres libres & $\alpha_{\mathrm{a}}$ & $\begin{array}{c}H_{\mathrm{e}, \mathrm{v}}^{\mathrm{c}} \\
{\left[\mathrm{W} / \mathrm{m}^{2} \mathrm{~K}\right]}\end{array}$ & \multicolumn{2}{|c|}{$\left[H_{\mathrm{a}, \mathrm{f}}^{\mathrm{c}}\right]$} & $\begin{array}{c}C_{\mathrm{i}} \\
{\left[\mathrm{J} / \mathrm{m}^{2} \mathrm{~K}\right]}\end{array}$ & $\begin{array}{c}C_{\mathrm{p}} \\
{\left[\mathrm{J} / \mathrm{m}^{2} \mathrm{~K}\right]}\end{array}$ & $\begin{array}{c}H_{\mathrm{i}} \\
{\left[\mathrm{W} / \mathrm{m}^{2} \mathrm{~K}\right]}\end{array}$ \\
\hline Valeur au minimum & 0,927 & 7 & 0,96 & 0,64 & 6900 & 6800 & 12,1 \\
\hline Erreur théorique & 0,11 & 4,6 & 0,89 & 0,92 & 3700 & 4800 & 5,6 \\
\hline Erreur positive & 0,097 & 5,3 & $>3,5$ & $>2$ & 4000 & 4700 & 6,5 \\
\hline Erreur négative & $-0,088$ & $-4,0$ & $-0,43$ & $-0,57$ & -3400 & -4600 & $-5,3$ \\
\hline
\end{tabular}




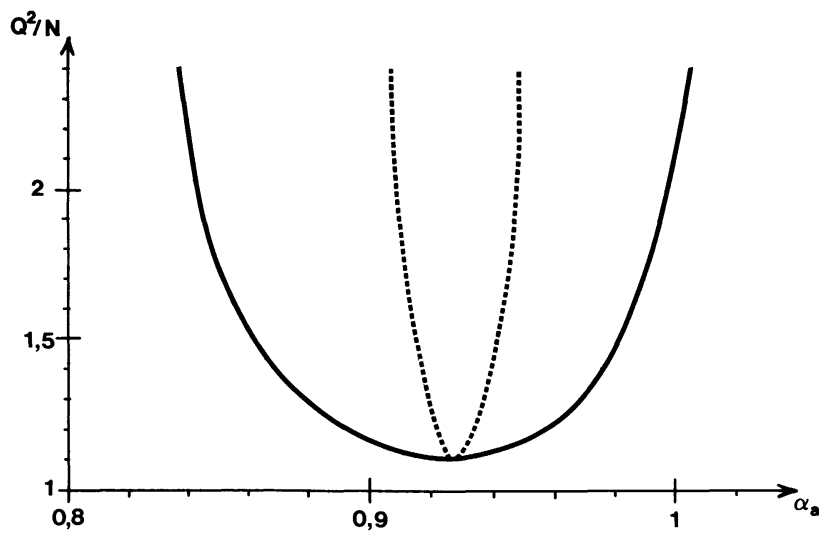

a)

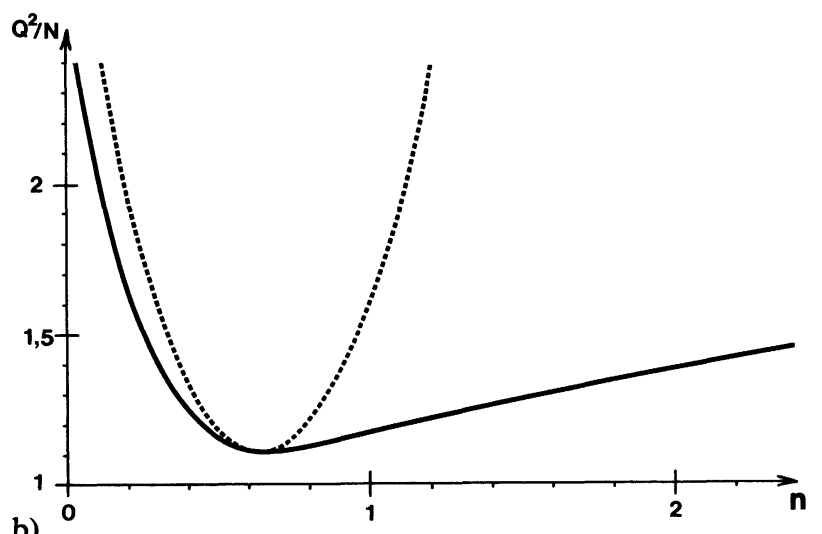

b)

Fig. 9. - Variation de l'écart quadratique $Q^{2} / N$ en fonction d'un paramètre libre ( $\alpha_{\mathrm{a}}$ courbe a, $n$ courbe b), tous les autres paramètres libres étant soit minimisés $(\longrightarrow)$, soit fixes (- - -).

[Weighted quadratic deviation $Q^{2} / N$ as a function of the value of one free parameter ( $\alpha_{\mathrm{a}}$ in $\mathrm{a}, n$ in $\mathrm{b}$ ), all other free parameters being fitted (-) or fixed at their terminal values (- - -).]

suivante $[2,18]$ : nous calculons la variation de $Q^{2}$ en fonction de chacun des paramètres libres, tous les autres paramètres libres étant ajustés par minimisation pour prendre en compte les corrélations paramétriques. Lorsque la variation de valeur du paramètre provoque un doublement de l'écart quadratique, nous obtenons la plage d'incertitude réelle sur ce paramètre ; l'effet de l'écart paramétrique sur $Q^{2}$ est alors égal à l'effet des incertitudes métrologiques.

Du tableau $\mathrm{V}$ et de la figure 9 on peut tirer deux observations principales:

- les incertitudes sur les paramètres $b$ et $n$ sont très dissymétriques; cela provient de la forte non linéarité du modèle vis-à-vis de ces deux paramètres ;

- les incertitudes sont dans l'ensemble très élevées ; cela découle de la très forte corrélation entre paramètres, précédemment évoquée. Les coefficients de corrélatîón calculés sont nettement surestimés à cause de la non linéarité du modèle. Notre "conviction intime", basée sur les recoupements entre quelques centaines de minimisations que nous avons effectuées sur cet exemple, est que l'incertitude physique des paramètres est largement inférieure à la valeur calculée.

7.1.4 Sensibilité des paramètres aux mesures aberrantes. - Pour que la procédure d'identification soit pertinente, il faut en particulier qu'elle soit insensible aux mesures aberrantes. Celles-ci sont définies comme étant les mesures pour lesquelles la valeur de $q_{j}^{2}$ (écart quadratique mesure-modèle) est supérieure à un certain seuil. Nous l'avons choisie ici égale à 8 ; la distribution de $q_{j}^{2}$ étant proche d'un $\chi^{2}(1)$, la probabilité théorique pour que $q_{j}^{2}$ soit supérieur à 8 est de $0,5 \%$.

Pour éprouver l'insensibilité des paramètres aux mesures aberrantes, nous avons ainsi effectué un ajustement avec coupure de $\chi^{2}$ : en partant de la valeur des paramètres au minimum, on effectue une minimisation en supprimant du calcul de $Q^{2}$ tous les événements pour lesquels $q_{j}^{2}$ est supérieur au seuil (ici 8).

Pour le cas traité, les résultats regroupés tableau VI illustrent bien l'insensibilité des paramètres aux mesures " coupées ». L'aspect aberrant de ces dernières est illustrée par deux résultats : d'une part les événements exclus sont peu nombreux

Tableau VI. - Valeurs des paramètres obtenues par minimisation avec et sans coupure de $\chi^{2}$; illustration de [Values of the fitted parameters with and without $\chi^{2}$ cut-off. Insensitivity of the parameters fitted values to the $x^{2}$ cut-off.]

\begin{tabular}{|c|c|c|c|c|c|c|c|}
\hline Paramètres libres & $\alpha_{\mathrm{a}}$ & $\begin{array}{c}H_{\mathrm{e}, \mathrm{v}}^{\mathrm{c}} \\
{\left[\mathrm{W} / \mathrm{m}^{2} \mathrm{~K}\right]}\end{array}$ & \multicolumn{2}{|c|}{$\left[H_{\mathrm{a}, \mathrm{f}}^{\mathrm{c}}\right]$} & $\begin{array}{c}C_{\mathrm{i}} \\
{\left[\mathrm{J} / \mathrm{m}^{2} \mathrm{~K}\right]}\end{array}$ & $\begin{array}{c}C_{\mathrm{p}} \\
{\left[\mathrm{J} / \mathrm{m}^{2} \mathrm{~K}\right]}\end{array}$ & $\begin{array}{c}H_{\mathrm{i}} \\
{\left[\mathrm{W} / \mathrm{m}^{2} \mathrm{~K}\right]}\end{array}$ \\
\hline Valeur au minimum & 0,927 & 7,4 & 0,96 & 0,64 & 6900 & 6800 & 12,1 \\
\hline $\begin{array}{l}\text { Valeur après ajustement } \\
\text { après coupure de } \chi^{2}\end{array}$ & 0,927 & 7,4 & 0,96 & 0,64 & 6900 & 7000 & 12,1 \\
\hline
\end{tabular}


Tableau VII. - Evolution de la valeur des paramètres libres en fonction du nombre de tranches élémentaires du modèle, dans le sens de l'écoulement.

[Parameters fitted value variation for the differents numbers of associated elementary models.]

\begin{tabular}{|c|c|c|c|c|c|c|c|c|}
\hline $\begin{array}{l}\text { Nombre de } \\
\text { tranches élémentaires }\end{array}$ & $\alpha_{\mathrm{a}}$ & $\begin{array}{c}H_{\mathrm{e}, \mathrm{v}}^{\mathrm{c}} \\
{\left[\mathrm{W} / \mathrm{m}^{2} \mathrm{~K}\right]}\end{array}$ & & $n$ & $\begin{array}{c}C_{\mathrm{i}} \\
{\left[\mathrm{J} / \mathrm{m}^{2} \mathrm{~K}\right]}\end{array}$ & $\begin{array}{c}C_{\mathrm{p}} \\
{\left[\mathrm{J} / \mathrm{m}^{2} \mathrm{~K}\right]}\end{array}$ & $\begin{array}{c}H_{\mathrm{i}} \\
{\left[\mathrm{W} / \mathrm{m}^{2} \mathrm{~K}\right]}\end{array}$ & $Q^{2} / N$ \\
\hline 1 & 0,944 & 7,5 & 0,69 & 0,68 & 6500 & 7600 & 12,0 & 1,5 \\
\hline 2 & 0,931 & 7,4 & 0,90 & 0,64 & 6800 & 7100 & 12,2 & 1,1 \\
\hline 3 & 0,928 & 7,4 & 0,95 & 0,65 & 6900 & 6900 & 12,1 & 1,1 \\
\hline 5 & 0,927 & 7,4 & 0,96 & 0,64 & 6900 & 6800 & 12,1 & 1,1 \\
\hline 10 & 0,927 & 7,4 & 0,96 & 0,64 & 6900 & 6800 & 12,1 & 1,1 \\
\hline
\end{tabular}

$(\simeq 2 \%)$ et, d'autre part, ils sont répartis de façon aléatoire tout au long des expériences.

\subsection{ANALYSE PHYSIQUE.}

7.2.1 Valeur des paramètres; interprétation physique. - En ce qui concerne la valeur des paramètres, il y a à la fois une incertitude sur la valeur attendue des paramètres (due au passage d'une grandeur locale à une grandeur intégrale) et une erreur sur la «mesure inclusive» du vecteur des paramètres.

Pour les coefficients d'absorption de la vitre et de l'absorbeur ainsi que pour les coefficients d'échange convectif, l'écart entre valeur attendue et mesurée n'excède pas l'incertitude a priori (Tab. III) : 5 à $10 \%$ pour les coefficients d'absorption, 10 à $20 \%$ pour les échanges convectifs.

La valeur de la capacité calorifique et celle de la conductivité de l'isolant constituant le fond du capteur sont dans la limite supérieure des valeurs attendues; le démontage a fourni une explication, en révélant la présence de défauts de polymérisation, difficulté signalée par le réalisateur du prototype.

7.2.2 Sensibilité des paramètres à une modification du modèle. - La méthode d'ajustement par minimisation de $\chi^{2}$ est robuste et opérationnelle pour les modèles non linéaires; par contre, l'interprétation des résultats purement statistiques est non directe. L'expertise physique est donc indispensable pour s'assurer de la pertinence des résultats obtenus.

L'évolution de la valeur des paramètres en fonction de modifications de structure du modèle nous parait être un critère important de pertinence physique. Nous présentons ici pour illustration la sensibilité des paramètres à la prise en compte des effets longitudinaux dans le capteur.

Nous avons modélisé le capteur avec un nombre croissant de "tranches " élémentaires, passant ainsi d'un modèle purement monodimensionnel à un modèle quasi-bidimensionnel assez complet. Les valeurs obtenues par minimisation pour chacun de ces modèles varient très régulièrement (Tab. VII). Les paramètres variant le plus sont ceux du coefficient d'échange convectif absorbeur-fluide; cela s'explique par l'approximation linéaire du profil de température du modèle à une tranche, qui est grossière, particulièrement pour les faibles débits [8]. Cette variation du coefficient d'échange s'associe à une variation - plus faible - du coefficient d'absorption et de la capacité calorifique de la peau (couplage entre phénomènes, corrélation entre les paramètres $\approx 0,6$ à 0,9 ).

7.2.3 Précision de la puissance extraite simulée. Domaine de validité du modèle. - La concordance puissance extraite mesurée-calculée pour les expériences utilisées lors de l'identification est très bonne puisqu'elle est à la limite de la précision instrumentale $[2,3]$. (La cohérence modèle-banc d'essai est donc satisfaite.)

Pour les expériences à faible débit (inférieur à $40 \mathrm{~m}^{3} / \mathrm{h}$ ), l'écart mesure-modèle est par contre plus élevé; la valeur du nombre de Reynolds pour ces débits $(\operatorname{Re}<2000)$ permet de suggérer une transition du régime d'écoulement conduisant à l'adoption d'une loi d'échange différente. Lorsque le débit d'air dans le capteur est variable, la concordance mesuremodèle est tout à fait satisfaisante. Les seules périodes où cet écart n'est plus négligeable sont celles où le débit est faible ; comme elles correspondent à de très faibles puissances fournies par le système, nous n'en avons pas tenu compte (cohérence modèle-objectifs).

Sur la figure 10, nous avons représenté les puissances extraites mesurée et calculée pour l'expérience la plus sévère ; malgré une dynamique particulièrement forte, la concordance mesure-modèle reste très bonne.

La bonne concordance mesure-modèle pour l'ensemble des expériences avec éclairement que nous avons réalisées a été présentée par ailleurs $[2,3]$. 

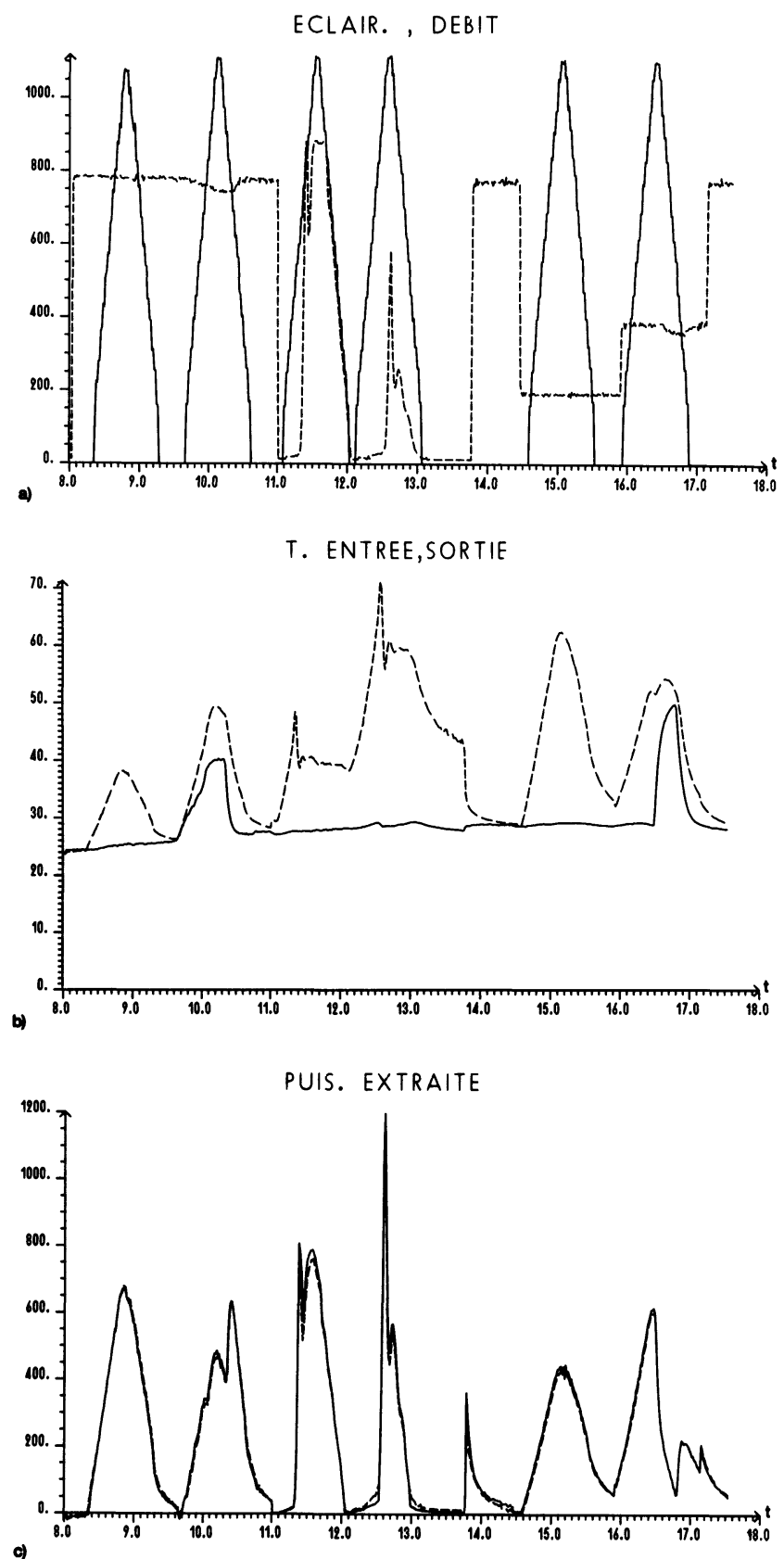

Fig. 10. - Variation de différentes grandeurs en fonction du temps (exprimé en heure), pour l'expérience «E.2» (éclairement, débit et température d'entrée d'air fortement 2 ,

masse $\times 10^{4}(\mathrm{~kg} / \mathrm{s})$; b) $(-)$ température d'entrée d'air dans le capteur ( $\left.{ }^{\circ}\right)$, (- - - -) température de sortie d'air ( $\left.{ }^{\circ}\right)$; c) (-) puissance thermique extraite calculée $\left(\mathrm{W} / \mathrm{m}^{2}\right),(---)$ puissance thermique extraite mesurée $\left(\mathrm{W} / \mathrm{m}^{2}\right)$.

[Time variation (hours) for the «E.2» experiment (the solar flux, the air flow rate and the inlet air temperature been strongly varying) a) (air mass flow rate $\times 10^{4}(\mathrm{~kg} / \mathrm{s})$, b) $(\longrightarrow)$ inlet temperature $\left({ }^{\circ} \mathrm{C}\right),\left(-\right.$ - $\left.^{-}\right)$outlet temperature ( $\left.{ }^{\mathrm{C}}\right)$, c) $(-)$ calculated extracted heat $\left(\mathrm{W} / \mathrm{m}^{2}\right),(---)$ measured extracted heat $\left(\mathrm{W} / \mathrm{m}^{2}\right)$.]

\section{Conclusion.}

Les quelques illustrations de mise en œuvre de la méthode présentée auront permis d'apprécier l'écart méthodologique séparant les expériences classiques de laboratoire et la caractérisation d'un composant industriel ; dans un cas on dispose d'un modèle de connaissance et on mesure et contrôle directement les grandeurs intervenant dans les phénomènes étudiés ; dans l'autre, la détermination du modèle fait partie intégrante du problème et on ne dispose que de quelques mesures globales ainsi que d'expériences ne découplant qu'imparfaitement les paramètres du modèle.

L'identification de modèles est un domaine en plein développement, tant en Thermique de l'Habitat [19 à 22] que plus généralement en Génie des Procédés et Systèmes Industriels [4, 23 à 28, 34]. Généralement issues du domaine de l'Automatique, diverses méthodes ont pu être utilisées pour la construction de modèles de commande et de régulation des processus industriels. Les modèles obtenus sont généralement des modèles mathématiques à partir desquels il est difficile de remonter aux valeurs physiques des paramètres.

Nous avons déjà largement montré la cohérence interne de notre approche; nous allons la situer par rapport à d'autres approches en ne nous intéressant qu'aux modèles à orientation physique, en régime dynamique :

L'intégration du facteur dynamique dans la métrologie elle-même permet dans les cas simples la détermination directe de paramètres physiques: citons par exemple la méthode «flash» pour la détermination d'une diffusivité thermique [29] ou une méthode en régime transitoire pour la mesure d'une chaleur spécifique fortement dépendante de la température [30].

A l'inverse, l'approche modale a été utilisée pour identifier les principales constantes de temps d'un système plus complexe réduit par analyse modale. On obtient alors un modèle à deux ou trois paramètres dynamiques sans qu'il soit possible de remonter aux grandeurs physiques du système complet $[19,20$, 28].

La réduction de modèle est généralement basée sur es operations statistiques proc es es no res, pour identifier un modèle réduit à un modèle de connaissance numériquement plus lourd [21, 27]. Dans ce cas, l'expérimentation est remplacée par une simulation de référence; l'établissement d'un modèle réduit est plus simple que pour l'identification d'un système réel car tous les phénomènes, décrits dans le modèle de connaissance, sont connus a priori.

L'identification d'un modèle physique réduit, avec prise en compte explicite des erreurs de mesures, a déjà été menée avec succès, il y a une dizaine 
d'années, pour l'étude thermique de systèmes réels (satellite, mur, échangeur) par filtrage de Kalman $[31,32]$. Le premier auteur signale cependant l'inadaptation de la méthode à la prise en compte de l'ensemble des données expérimentales dynamiques. Le filtrage de Kalman semble plus adapté à l'identification récursive de systèmes linéaires à paramètres lentement variables; il resterait cependant à la confronter aux moindres carrés récursifs.

En définitive, ce qui nous semble déterminant dans la méthode décrite ici, c'est la construction d'une métrique de l'espace de travail paramétrique directement à partir des incertitudes métrologiques. Elle permet une utilisation fine de la réponse dynamique du système aux diverses expériences. Alors, la sensibilité des paramètres, le choix des paramètres libres, les incertitudes paramétriques peuvent être définis rigoureusement.

En conclusion, la méthode présentée a tenté d'étendre la possibilité de caractérisations dynamiques de modèles, lors de la présence de phénomènes couplés nécessitant la détermination d'un nombre élevé de paramètres. Nous avons pu illustrer sa pertinence :

- pour la détection d'aberrations métrologiques ou d'inadéquation du modèle ;

- pour la détection de défauts dans la fabrication du composant testé ;

- pour la critique physique de la valeur des paramètres et de leur nombre ;

- pour la détermination des incertitudes et du domaine de validité du modèle réduit.

En revanche, elle repose sur une démarche nécessairement minutieuse :

- de par l'importance de la détermination des erreurs instrumentales ;

- par l'utilisation différenciée de nombreuses séquences expérimentales ;

- de par la difficulté d'interpréter les résultats statistiques agrégés pour les modèles non linéaires.

Enfin, le coût de mise en ouvre informatique est relativement important. Signalons pour terminer que nous avons pu utiliser la même démarche avec succès pour un système très différent de celui qui nous a, dans cet article, servi à l'illustrer (stockage souterrain de gaz [33]).

\section{Annexe}

Propagation des erreurs de mesures.

Pour le critère d'ajustement que nous avons choisi, l'erreur de mesure $\varepsilon_{\mathrm{i}}$ de la différence puissance extraite mesurée-calculée doit être estimée précisément à chaque instant. Dans le cas traité ici, la distribution de $\varepsilon_{\mathrm{i}}$ est variable d'une part parce que le modèle du capteur étudié est non linéaire et, d'autre part, parce que les écarts types des disributions des erreurs de mesures ne sont pas tous constants : l'erreur sur la mesure du débit et des températures entrée-sortie de l'air dans le capteur dépend de la valeur du débit; l'erreur sur la mesure du flux est une fonction de cette grandeur. L'erreur $\varepsilon_{j}$ s'exprime de la façon suivante :

$$
\varepsilon_{j}=\delta\left(\mu_{j}-M_{j}\right)=\delta\left(\dot{m} \cdot C_{\mathrm{p}} \cdot\left[\theta_{j}-T_{j}\right]\right)
$$

avec

$\mu_{j}$ : puissance extraite mesurée

$M_{j}:$ puissance extraite calculée

$\dot{m}:$ débit-masse

$\theta_{j}:$ température de sortie d'air mesurée

$T_{j}$ : température de sortie d'air calculée

Dès que la différence entre température de sortie mesurée-calculée est faible et que les erreurs sont indépendantes, on a :

$$
\varepsilon_{j} \approx \dot{m} \cdot C_{\mathrm{p}} \sqrt{\left(\delta \theta_{j}^{2}+\delta T_{j}^{2}\right)} .
$$

L'erreur de mesure $\delta \theta_{j}$ de la température de sortie est estimée directement et nous présentons cidessous une procédure possible pour la détermination de $\delta T_{j}$. La température de sortie $T_{j}$, à l'instant $j$, peut s'exprimer en fonction de l'ensemble des valeurs des grandeurs d'entrée depuis l'instant 0 (pour lequel l'état du capteur est supposé connu) à l'instant $j$ :

$$
T_{j}[\mathbf{E}(j), \mathbf{E}(j-1), \ldots, \mathbf{E}(0)]
$$

avec $\mathbf{E}(n)$, vecteur des grandeurs d'entrée à l'instant $n$.

Une erreur de mesure $\delta \mathbf{E}$ des grandeurs d'entrée à l'instant $n$ induira une erreur $\delta T_{j}(n, \delta \mathbf{E})$ sur la température de sortie calculée à l'instant $j$ :

$$
\begin{array}{r}
\delta T_{j}(n, \delta \mathbf{E})=T_{j}[\mathbf{E}(j), \ldots, \mathbf{E}(n)+\delta \mathbf{E}, \ldots, \mathbf{E}(0)]- \\
-T_{j}[\mathbf{E}(j), \ldots, \mathbf{E}(0)] .
\end{array}
$$

Les erreurs de chacune des grandeurs d'entrée étant indépendantes entre elles, l'écart type de la distribution de $T_{j}$ en fonction des erreurs de mesure des entrées à l'instant $n$ s'écrit au premier ordre :

$$
\delta T_{j}(n)=\sqrt{\sum_{m=1}^{k}\left[\delta T_{j}\left(n, \boldsymbol{\sigma}\left(E_{\mathrm{m}}\right)\right)\right]}
$$

avec

$k:$ nombre des grandeurs d'entrée

$\boldsymbol{\sigma}\left(E_{\mathrm{m}}\right)$ : écart type de l'erreur de mesure de la grandeur d'entrée $E_{\mathrm{m}}$.

Les erreurs de mesure étant temporellement indépendantes, la propagation à l'instant $j$ de l'ensemble des erreurs des grandeurs d'entrée de l'instant initial 
0 à l'instant $j$ nous permet de calculer l'écart type de la distribution de $T_{j}$ :

$$
\delta T_{j}=\sqrt{\sum_{n=0}^{i}\left[\delta T_{j}(n)^{2}\right]} .
$$

Ces erreurs ont ainsi été calculées par tirage à chaque instant $j$. Pour des raisons évidentes de temps de calcul, nous n'avons pas propagé l'ensemble des erreurs depuis l'instant initial, mais avons utilisé un horizon fuyant, de durée variable, dépendant du débit.

Outre l'ensemble des erreurs des grandeurs d'entrée, nous avons également pu de la même manière propager l'incertitude de l'état initial du capteur. On constate que cette dernière ne devient négligeable qu'après $1 \mathrm{~h}$ environ alors qu'un horizon de 5 min suffit en moyenne à gommer les erreurs courantes.

\section{Bibliographie}

[1] Présentation du projet ALMETH, Session National Projects, Proceedings of the Workshop on Future Building Energy Modelling, Ispra (Italie), Ed. D. Van Hattem, Joint Research Center, Ispra (1987) pp. 167-216.

[2] Dufresne J. L., Etude et développement d'une procédure expérimentale pour l'identification des paramètres d'un modèle thermique de capteurs solaires à air en régime dynamique, Thèse de Doctorat, Université Paris VII, juin 1987.

[3] Dufresne J. L., Chounet L. M., PiCARd D., GailLARD P., NopPe J. M., Présentation d'une procédure expérimentale pour la caractérisation d'un composant en régime dynamique : application à un mur-capteur solaire à air, Rev. Gen. Therm. 339 (1990) 135-151.

[4] Filippi C., GrefFe J. L., Bordet J., VillermauX J., Barnay J. L., Bonte P., Georgakis K., Tendency modeling of semibatch reactors for optimization and control, Chem. Eng. Sci. 41 (1986) 913-922.

[5] Eadie W. T., Drijard D., James F. E., Roos M., SADOULET B., Statistical methods in experimental physics (North-Holland Ed., Amsterdam) 1982.

[6] TAupIN D., Probabilities data reduction and error analysis in the physical sciences (Les Editions de Physique, Les Ulis, France) 1988.

[7] Auger J. L., Alexandre A., Martinet J., Fonctionnement de capteurs solaires plans en régime variable, Rev. Gen. Therm. 239 (1981) 811-824.

[8] Rivet P., Analyse élémentaire de l'équation simplifiée du capteur-plan à air en régime stationnaire, Revue Phys. Appl. 15 (1980) 977-983.

[9] LeTZ T., LALlemand M., Etude théorique et expérimenta e un capteur so aire $\mathrm{p}$ an a air en regime dynamique, Revue Phys. Appl. 21 (1986) 727734.

[10] FiSCH N. M., Systemuntersuchungen zur nutzung der Sonnenenergie bei der Beheizung von Wohngebäuden mit Luft als Wärmeträger, Thèse de Docteur Ingénieur, Institut für Thermodynamik und Wärmetechnik, Universität Stuttgart (R.F.A.) 1984.

[11] Dufresne J. L., Lakhsassi A., Lahellec A., PICARD D., Problème de commande d'un champ de capteur solaire à air en climat tempéré (semiocéanique), Comptes rendus des $2^{\text {ndes }}$ Journées internationales de Thermique (JITH-85), Rabat, 2-5 avril 1985, Ed. CNRS (PIRSEM) 1986, pp. 1035-1045.

[12] MCAdams W. H., Transmission de la chaleur (Ed. Dunod) 1961.

[13] Kays W. M., Crawford M. E., Convective heat and mass transfer (McGraw-Hill Book Company Ed.) 1980 .

[14] Bevington P. R., Data reduction and error analysis for the Physical Sciences (McGraw-Hill Ed.) 1969.

[15] JAMES F., Roos M., MINUIT : a system for function minimization and analysis of the parameters errors and correlations (Document D 506, CERN Computer Center program Library, Edition CERN, Genève, Suisse) 1983.

[16] JAMES F., Function minimization, Proceedings of the 1972 CERN computing and data processing school (Document CERN 72-21, Edition CERN, Genève, Suisse) 1972.

[17] LAKHSASSI A., Etude expérimentale de capteurs solaires à air, en climat océanique, Thèse de Doctorat, Université Paris VII, Octobre 1983.

[18] JAMES F., Interpretation of the errors on parameters as given by MINUIT (CERN Computer Center Program Library, Edition CERN, Genève, Suisse), 1978.

[19] Neveu A., Bacot P. et Regas R., Modèle d'évolution thermique des bâtiments. Conditions pratiques d'identification, Rev. Gen. Therm. Fr. 296297 (1986) 413-420.

[20] NeIRAC F. P., Approche théorique et expérimentale des modèles réduits du comportement thermique des bâtiments. Thèse de l'Ecole des Mines de aris, specia ite nergetique mars

[21] Benard C., Guerrier B., Mahmalji A., Estimation de paramètres ou de conditions limites d'un système thermique, à l'aide de différentes techniques d'optimisation. Compte rendu de la journée d'étude "estimation des paramètres et problèmes inverses en conduction ", mai 1986, édité par la Société Française des Thermiciens.

[22] SPIRKL W., Parameter identification and model choosing by Bayesian inference with application to solar energy. Proceedings of the ISES-Solar World Congress, Hamburg (R.F.A.) 13-18 septembre 1987 (Edition Pergamon Press). 
[23] LANDAU I. D., Identification et commande des systèmes (Editions Hermes) 1988.

[24] Landau I. D., M'SAAd M., M'Sirdi N. K., CANUDAS C., Identification d'un échangeur à l'aide d'un Apple II, Rev. Gen. Therm. Fr. 280 (1985) 49-55.

[25] Fort C., SAulnier J. B., Méthodes nodale et estimation de paramètres; Compte rendu de la journée d'étude «estimation des paramètres et problèmes inverses en conduction (mai 1986) édité par la Société Française des Thermiciens.

[26] Chillet Ch., Hadi A. W., Perret R., Isnard R., Identification $\mathrm{du}$ modèle thermique d'une machine à induction de puissance moderne, Revue Phys. Appl. 24 (1989) 923-931.

[27] MAYe J. P., Legrand S., Identification par fonction de transfert d'un accumulateur à chaleur lente de type tubulaire, Rev. Gen. Therm. Fr. 277 (1985) 41-47.

[28] Pasquetti R., Petit D., Analyse modale d'un processus de diffusion thermique : identification par thermographie infra-rouge, Int. J. Heat Mass Transfer. 31 (1988) 487-496.

[29] Degiovanni A., Une nouvelle technique d'identification de la diffusivité thermique par la méthode "flash", Revue Phys. Appl. 21 (1986) 229-237.

[30] Jarny Y. C., Delaunay D. M., Application de la méthode O.L.S.M. à la détermination d'une chaleur spécifique fortement variable en fonction de la température. Compte rendu de la journée d'étude "estimation des paramètres et problèmes inverses en conduction ", mai 1986, édité par la Société Française des Thermiciens.

[31] SAUlNiER J. B., La modélisation thermique et ses applications aux transferts couplés et au contrôle actif. Thèse de Doctorat ès Sciences Physiques, Université de Poitiers (Octobre 1980).

[32] Benolol Chocron S., Mise en place d'une technique d'identification paramétrique et de correction automatisée de modèles thermiques en régime stationnaire. Application à un radiateur à ailettes air-eau, Thèse de Docteur Ingénieur spécialité énergétique, Université de Poitiers (Octobre 1982)

[33] Dufresne J. L., Chounet L. M., Lahellec A., Développement d'un outil pour l'identification de modèles physiques réduits : application à un modèle simple de stockage souterrain de gaz, Rapport de contrat entre l'équipe RAMSES (SPI-CNRS) et le DAS (DETN-GDF) novembre 1989.

[34] Mishra S. and Parker J. C., Parameter estimation for coupled unsaturated flow and transport, Water Resources Res. 25 (1989) 385-396. 